\title{
Minimax particle filtering for tracking a highly maneuvering target
}

\author{
Jaechan Lim ${ }^{1,2} \mid$ Hun-Seok Kim ${ }^{1} \mid$ Hyung-Min Park ${ }^{2}$ (D)
}

\author{
${ }^{1}$ Department of Electrical Engineering \\ and Computer Science, University of \\ Michigan, Ann Arbor, Michigan \\ ${ }^{2}$ Department of Electronic Engineering, \\ Sogang University, Seoul, South Korea

\section{Correspondence} \\ Hyung-Min Park, Department of \\ Electronic Engineering, Sogang \\ University, Seoul 04107, South Korea. \\ Email: hpark@sogang.co.kr

\section{Funding information} \\ Basic Science Research Program, \\ Grant/Award Number: \\ NRF-2016R1D1A1A09918304 and \\ NRF-2019R1I1A1A01058976
}

\begin{abstract}
Summary
In this paper, we propose a new framework of particle filtering that adopts the minimax strategy. In the approach, we minimize a maximized risk, and the process of the risk maximization is reflected when computing the weights of particles. This scheme results in the significantly reduced variance of the weights of particles that enables the robustness against the degeneracy problem, and we can obtain improved quality of particles. The proposed approach is robust against environmentally adverse scenarios, particularly when the state of a target is highly maneuvering. Furthermore, we can reduce the computational complexity by avoiding the computation of a complex joint probability density function. We investigate the new method by comparing its performance to that of standard particle filtering and verify its effectiveness through experiments. The employed strategy can be adopted for any other variants of particle filtering to enhance tracking performance.
\end{abstract}

\section{KEYWORDS}

bearing, minimax, particle filtering, range, risk, target tracking

\section{1 | INTRODUCTION}

Conventional adaptive filters such as the adaptive Kalman filter can be applied to maneuvering target tracking. ${ }^{1}$ In this adaptive filter, two additional schemes exist beyond the standard Kalman filtering, ie, maneuvering detection and adjusting the state noise variance. This adaptive filter can be applied to the problems of tracking a maneuvering target by white-noise acceleration models. More recently, the interactive multiple model extended Kalman filter (IMM-EKF) was proposed for tracking maneuvering targets where the state model varies based on multiple constant turn models. Moreover, IMM-EKF has been successfully applied for tracking a maneuvering target across various problems. Particle filtering (PF) was applied in the form of IMM that outperformed conventional IMM-EKF. ${ }^{2,3}$

In addition, PF was applied for solving this highly nonlinear estimation problem where the particles are used to approximate the probability density function because we cannot compute the expected state with respect to the posterior function in a closed-form; PF has demonstrated powerful tracking performance in various dynamic state estimation problems, particularly when the state system and measurement functions are nonlinear functions with respect to the "state." Since its initial implementations in the 1990s, PF has become even more powerful owing to significant advancements in computing processors that enabled practical implementations in various problems ${ }^{4,5}$ Furthermore, various variants of PF have been proposed since its initial proposal such as auxiliary PF (APF), ${ }^{6}$ regularized PF (RPF), ${ }^{7}$ Kullback-Leibler divergence PF (KLDPF), ${ }^{8-10}$ Gaussian PF (GPF), ${ }^{11,12}$ and cost-reference PF. ${ }^{13-15}$ The APF algorithm is the same as that of sequential importance resampling PF (SIRPF) until the resampling process; thereafter, we go back to the previous time step and propagate the particles again based on resampled particles. Standard PF (SPF) 
undergoes its inherent defect of particle impoverishment, and RPF was particularly modified from SPF to overcome the problem. The primary feature of KLDPF is that the number of employed particles is optimized adaptively based on a predefined error bound at every time step. In addition, GPF comprises a simple algorithm with excellent performance that does not require resampling, which makes GPF robust against particle impoverishment phenomenon that results from the resampling process. Cost-reference PF is applied with unknown noise statistical information and is robust against particle impoverishment because the employed proposal density is based on a Gaussian function as in GPF.

Theoretically, PF can show optimal performance with infinitely many particles in nonlinear problems, and outperforms various suboptimal approaches such as the extended Kalman filter and Kalman variants, with a reasonable number of particles. Nevertheless, occasionally, we are not able to obtain satisfactory results by PF when the time-varying target state varies drastically and highly maneuvers. One crucial factor for the nonideal performance of PF is that degeneracy problem; that is, after a few iterations, we have only one particle that has meaningful weight while all the other particles have almost zeros weights, and the variance of the weights only increases over time that eventually results in unsatisfactory performance of $\mathrm{PF}^{16}$ To get over with the degeneracy problem, the resampling process is adopted that regenerate high-quality particles more often; nonetheless, we also have a side effect of particle impoverishment by resampling that we may have all the same particles within a few iterations, particularly when we have very small state noise. Therefore, the variance of the weights of particles is crucial to obtain the successful performance of PF approaches. To this end, we adopt a minimax strategy by which we can obtain significantly reduced the variance of the weights of particles that eventually results in robustness against maneuvering target and improved tracking performance of PF approaches. In this framework, we maximize a predefined risk function on the condition that the risk is bounded over time; subsequently, we obtain the estimator that minimizes the maximized risk function. Therefore, the bounded maximum risk is minimized in this strategy to avoid the worst-case divergence from true trajectories and provides more robust performance. In this strategy, the focus is directed to avoiding large errors rather than merely minimizing errors in the problem where large errors exist due to large variations in the state. To accomplish this goal, we follow the same criterion of minimum mean square error (MMSE) while using the possible maximum MSE as a risk function to be minimized.

In this paper, we propose a new algorithm of minimax-PF (MPF), especially for tracking problems where a target state is highly maneuvering. We show the outperforming results of the proposed MPF compared with the standard PF. Further, we employ the minimax strategy to IMM-PF and show its outperforming result over conventional IMM-EKF and IMM-PF. The proposed approach can be adopted for any variants of PF to improve their tracking performances as shown in the experiments.

This paper is organized as follows. In Section 2, we describe the problem formulation. In Section 3, we describe the proposed MPF. In Section 4, we assess the performance of the proposed MPF compared to nonminimax particle filers; besides, we apply the minimax strategy to IMM-PF and compare the performances of IMM-EKF, IMM-PF, and IMM-MPF. Finally, we conclude and provide remarks in the last section.

\section{2 | PROBLEM FORMULATION}

In this problem, we track the location and velocity of a single target in a two-dimensional space where a target is moving with random acceleration based on range and bearing measurements. This problem is of interest in various applications such as radar systems and is a paradigm for target tracking problems. We estimate the state of the target based on observations measured at the origin of the coordinate system, as shown in Figure 1A. The moving direction of the target is subject to the acceleration that is determined by the process noise in the state equation. We denote the state and measurement by $\theta$ and $\boldsymbol{z}$, respectively, and the state equation is expressed as follows ${ }^{17-19}$ :

$$
\underbrace{\left[\begin{array}{c}
r_{x, k} \\
r_{y, k} \\
v_{x, k} \\
v_{y, k}
\end{array}\right]}_{\boldsymbol{\theta}_{k}}=\underbrace{\left[\begin{array}{llll}
1 & 0 & T & 0 \\
0 & 1 & 0 & T \\
0 & 0 & 1 & 0 \\
0 & 0 & 0 & 1
\end{array}\right]}_{\boldsymbol{A}_{1}} \underbrace{\left[\begin{array}{l}
r_{x, k-1} \\
r_{y, k-1} \\
v_{x, k-1} \\
v_{y, k-1}
\end{array}\right]}_{\boldsymbol{\theta}_{k-1}}+\boldsymbol{A}_{2} \boldsymbol{u}_{k},
$$


FIGURE 1 Target is tracked based on range $R$ and bearing $B$ in radar [Colour figure can be viewed at wileyonlinelibrary.com]

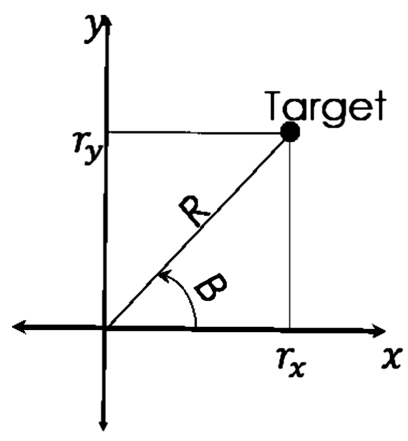

(A)

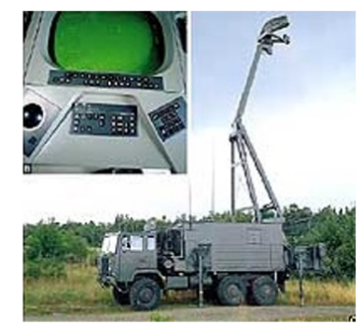

(B)

where

$$
\boldsymbol{A}_{2}=\left[\begin{array}{cc}
\frac{T^{2}}{2} & 0 \\
0 & \frac{T^{2}}{2} \\
T & 0 \\
0 & T
\end{array}\right], \quad \boldsymbol{u}_{k}=\left[\begin{array}{l}
u_{x, k} \\
u_{y, k}
\end{array}\right] .
$$

$r, v, u$, and $(x, y)$ denote the location, velocity, acceleration, and coordinates, respectively. $T$ is the sampling period, and $k$ is the discrete-time index. Therefore, the time-varying state is composed of four elements, ie, 2D location and 2D velocity. The state of the location and the velocity are subjected to a random process of $\boldsymbol{u}_{k}$. The range and bearing compose the measurement equation, which is highly nonlinear, and described as follows:

$$
\boldsymbol{z}_{k}=\boldsymbol{f}\left(\boldsymbol{\theta}_{k}\right)+\boldsymbol{\epsilon}_{k}=\left[R_{k} B_{k}\right]^{\top}+\boldsymbol{\epsilon}_{k},
$$

where

$$
\boldsymbol{z}_{k}=\left[z_{1, k} z_{2, k}\right]^{\top}, \boldsymbol{f}\left(\boldsymbol{\theta}_{k}\right)=\left[f_{1}\left(\boldsymbol{\theta}_{k}\right) f_{2}\left(\boldsymbol{\theta}_{k}\right)\right]^{\top},
$$

the range

$$
z_{1, k}=z_{R, k}=R_{k}+\epsilon_{R, k}=\sqrt{r_{x, k}^{2}+r_{y, k}^{2}}+\epsilon_{R, k},
$$

the bearing

$$
z_{2, k}=z_{B, k}=B_{k}+\epsilon_{B, k}=\arctan 2\left(r_{y, k}, r_{x, k}\right)+\epsilon_{B, k},
$$

and the measurement noise $\epsilon_{k}=\left[\epsilon_{R, k} \epsilon_{B, k}\right]^{\top}$. $\arctan 2\left(r_{y, k}, r_{x, k}\right)$ denotes the four-quadrant inverse tangent that acts on $r_{y, k}$ and $r_{x, k}$ elementwise to return $B_{k}$. We assume that a measurement noise $\epsilon_{i}$ for the measurement $z_{i, k}$ follows a zero-mean white Gaussian

$$
\epsilon_{i, k} \sim \mathcal{N}\left(0, \sigma_{i, k}^{2}\right) \text { for } i=1, \ldots, M,
$$

where $M$ is the total number of employed measurements. In this case, the likelihood function with respect to only $z_{i, k}$ becomes

$$
p\left(z_{i, k} \mid \boldsymbol{\theta}_{k}\right)=\frac{1}{\sqrt{2 \pi \sigma_{i, k}^{2}}} \exp \left\{-\frac{\left[z_{i, k}-f_{i}\left(\boldsymbol{\theta}_{k}\right)\right]^{2}}{2 \sigma_{i, k}^{2}}\right\}
$$

\section{3 | PROPOSED APPROACH}

\section{1 | Minimax approach}

In game theory, a minimax approach is employed as a solution for zero-sum game problems..$^{20,21}$ We model the tracking problem as a game where one player is the estimator that tries to obtain the accurate values of the time-varying state whereas the other player is the environment that adds the noise to the state and measurements to disturb the other 
player estimating the state. In this approach, the cost function is designed based on the strategy that the probability of the maximum expected point-loss is minimized regardless of the strategy of the opponent. Therefore, the expected point-loss becomes the risk that is maximized before the minimization in the minimax strategy. The minimax approach minimizes its maximal risk among all estimators, which can be described as follows:

$$
\inf _{\hat{\theta}_{k}} \sup _{\epsilon_{k}} \mathcal{R}_{k}\left(\theta_{k}, \hat{\theta}_{k}\right)
$$

where "inf," "sup," and $\mathcal{R}$ denote "infimum," "supremum," and risk, respectively.

\subsection{Minimax particle filtering (MPF)}

In the MMSE criterion of Bayesian estimation, the following mean square error is defined as the risk function to be minimized:

$$
\int\left(\boldsymbol{\theta}_{k}-\hat{\boldsymbol{\theta}}_{k}^{\mathrm{MMSE}}\right)^{2} p\left(\boldsymbol{\theta}_{0: k} \mid \boldsymbol{z}_{1: k}\right) \mathrm{d} \boldsymbol{\theta}_{k}
$$

to obtain the following MMSE:

$$
\hat{\boldsymbol{\theta}}_{k}^{\mathrm{MMSE}}=\int \boldsymbol{\theta}_{k} p\left(\boldsymbol{\theta}_{0: k} \mid \boldsymbol{z}_{1: k}\right) \mathrm{d} \boldsymbol{\theta}_{k},
$$

where the square error, $\left(\boldsymbol{\theta}_{k}-\hat{\boldsymbol{\theta}}_{k}^{\mathrm{MMSE}}\right)^{2}$ is defined as the cost function in this MMSE estimator. Similarly, in PF,

$$
\mathcal{R}_{k}^{\mathrm{PF}}=\sum_{j_{p}=1}^{N}\left[\left(\boldsymbol{\theta}_{k}^{j_{p}}-\hat{\boldsymbol{\theta}}_{k}^{\mathrm{PF}}\right)^{2} \omega_{k}^{j_{p}}\right], \text { where } \sum_{j_{p}=1}^{N} \omega_{k}^{j_{p}}=1
$$

is the risk function that we minimize, where $N$ is the number of employed particles, $j_{p}$ is the particle index, $\omega_{k}^{j_{p}}$ is the weight of the particle $j_{p}$ at time step $k$, and $\boldsymbol{\theta}_{k}^{j_{p}}$ is the particle with the index $j_{p}$; therefore, we obtain

$$
\hat{\boldsymbol{\theta}}_{k}^{\mathrm{PF}}=\sum_{j_{p}=1}^{N} \omega_{k}^{j_{p}} \boldsymbol{\theta}_{k}^{j_{p}}
$$

because

$$
\frac{\partial \mathcal{R}_{k}^{\mathrm{PF}}}{\partial \hat{\boldsymbol{\theta}}_{k}^{\mathrm{PF}}}=-2 \sum_{j_{p}=1}^{N} \boldsymbol{\theta}_{k}^{j_{p}} \omega_{k}^{j_{p}}+2 \hat{\boldsymbol{\theta}}_{k}^{\mathrm{PF}} \sum_{j_{p}=1}^{N} \omega_{k}^{j_{p}},
$$

and (13) makes (14) zero.

To formulate the minimax strategy for PF, from (12), we describe a new risk function with respect to each particle as follows:

$$
\mathcal{R}_{k}^{\mathrm{MPF}}=\sum_{j_{p}=1}^{N} \mathcal{G}\left(\mathcal{R}_{k}^{\mathrm{MPF}, j_{p}}\right)=\sum_{j_{p}=1}^{N}\left[\left(\boldsymbol{\theta}_{k}^{j_{p}}-\hat{\boldsymbol{\theta}}_{k}^{\mathrm{MPF}}\right)^{2} \omega_{k}^{j_{p}}\right] .
$$

In the proposed minimax-PF, we adopt a minimax strategy to the computations of the weights of particles. In particular, we select only one measurement that may incur the highest risk. That is, for $M$ measurements, we use only use one measurement that provides the minimum weight rather than the maximum weight. Therefore, to apply this approach, multiple measurements are required; otherwise, the approach becomes identical to that of regular PF.

According to (15), only $\omega_{k}^{j_{p}}$ is the factor that affects the magnitude of the risk, and the weight can be associated with a measurement as follows:

$$
\omega_{k}^{j_{p}} \in\left\{\omega_{1, k}^{j_{p}}, \omega_{2, k}^{j_{p}}, \ldots, \omega_{M, k}^{j_{p}}\right\}
$$

where, for example, $\omega_{1, k}^{j_{p}}$ is the weight of the particle $\boldsymbol{\theta}_{k}^{j_{p}}$ computed based on only the measurement $z_{1, k}$. The argument $i$ is determined with respect to each particle. Therefore, in minimax-PF, we maximize the risk with respect to each 
TABLE 1 Algorithm of minimax particle filtering $(\mathrm{PF})$ for sequential importance resampling PF. Only the steps, which are boldfaced, ie, (b) $\sim(d)$, of computing the weights of particles are different from those of standard PF

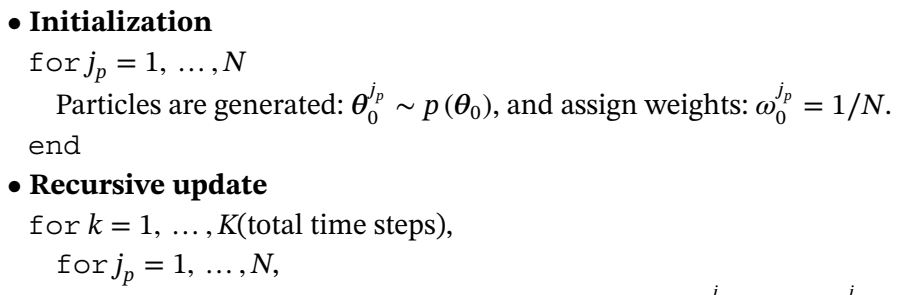

(a) Generate particles from a proposal density $q(\cdot): \boldsymbol{\theta}_{k}^{j_{p}} \sim q\left(\boldsymbol{\theta}_{k} \mid \boldsymbol{\theta}_{k-1}^{j_{p}}, \boldsymbol{z}_{k}\right)$.

(b) Compute the weights with respect to each measurement: $\omega_{i, k}^{j_{p}}=p\left(z_{i, k} \mid \boldsymbol{\theta}_{k}^{j_{p}}\right)$ for $i=1, \ldots, M$ assuming we employ prior density, $q\left(\boldsymbol{\theta}_{k} \mid \boldsymbol{\theta}_{k-1}^{j_{p}}, \boldsymbol{z}_{k}\right)=p\left(\boldsymbol{\theta}_{k} \mid \boldsymbol{\theta}_{k-1}^{j_{p}}\right)$, as the proposal density. end for $j_{p}=1, \ldots, N$,

(c) Normalize the weights with respect to each measurement: $\bar{\omega}_{i, k}^{j_{p}}=\frac{\omega_{i, k}^{j_{p}}}{\sum_{j_{p}=1}^{N} \omega_{i, k}^{j_{p}}}$ for $i=1, \ldots, M$.

(d) Select the minimum weight among $M$ weights for each particle:

$$
\omega_{k}^{j_{p}}=\bar{\omega}_{i_{p}, k}^{j_{p}} \text {, where } i_{p}=\underset{i \in\{1, \ldots, M\}}{\operatorname{argmin}} \bar{\omega}_{i, k}^{j_{p}} .
$$

end

(e) Normalize the weights: $\bar{\omega}_{k}^{j_{p}}=\frac{\omega_{k}^{j_{p}}}{\sum_{j_{p}=1}^{N} \omega_{k}^{j_{p}}}$ for $j_{p}=1, \ldots, N$.

(f) Compute the estimate: $\hat{\boldsymbol{\theta}}_{k}^{\mathrm{MPF}}=\sum_{j_{p}=1}^{N} \bar{\omega}_{k}^{j_{p}} \boldsymbol{\theta}_{k}^{j_{p}}$.

(g) Resample the particles. ${ }^{16}$ end

particle by associating the minimum weight to a particle among $M$ weights. Subsequently, we maximize the following total risk:

$$
\mathcal{R}_{k}^{\mathrm{MPF}, \mathrm{Max}}=\sum_{j_{p}=1}^{N}\left[\left(\boldsymbol{\theta}_{k}^{j_{p}}-\hat{\boldsymbol{\theta}}_{k}^{\mathrm{MPF}}\right)^{2} \omega_{i_{p}, k}^{j_{p}}\right],
$$

where the association between the measurement index $i_{p}$ and the particle $j_{p}$ is determined by

$$
i_{p}=\underset{i \in\{1, \ldots, M\}}{\operatorname{argmin}} \omega_{i, k}^{j_{p}}
$$

and the estimate of MPF at time step $k$

$$
\hat{\boldsymbol{\theta}}_{k}^{\mathrm{MPF}}=\sum_{j_{p}=1}^{N} \omega_{i_{p}, k}^{j_{p}} \boldsymbol{\theta}_{k}^{j_{p}} .
$$

At every time step, for the computation of the weight of every single particle, we may select a different single measurement that devalues the particle as low as possible.

The MPF algorithm is summarized in Table 1 where only the boldfaced steps, ie, $(b) \sim(d)$, of computing the weights of particles are different from those of a standard PF. Applying the proposed minimax strategy to any variants of PF is straightforward because it only requires modifying the step of computing the weights of particles.

\section{PERFORMANCE ASSESSMENT}

In this section, we assess the performance of the proposed minimax-PFs by comparing to that of regular PFs. We consider SIRPF first for a regular PF. Regarding the state noises, we first specify a parameter $\xi$ and randomly generate a noise variance to be “ $\xi \cdot V(0,1)$," where $\mathcal{V}(0,1)$ is the standard uniform distribution. Subsequently, random noise is generated by a Gaussian distribution that has zero mean with the generated variance of $\xi \cdot \mathcal{V}(0,1)$. We performed experiments with two kinds of $\xi$ in terms of magnitude to represent low and high maneuvering targets, respectively. We denote scenarios by $S_{S}$ and $S_{L}$ for small and large values of $\xi$, respectively. Figure 2 shows an illustrative example of two trajectories of a target where two significantly different magnitudes of $\xi$ are employed. With $\xi=10$ for the state noise, the trajectory of the target labeled as $T-1$ shows highly maneuvering. On the contrary, a run with $\xi=0.1$ results in the trajectory labeled as $T$ - 2 that shows significantly less maneuvering compared to $T$ - 1 during the same elapsed time. 

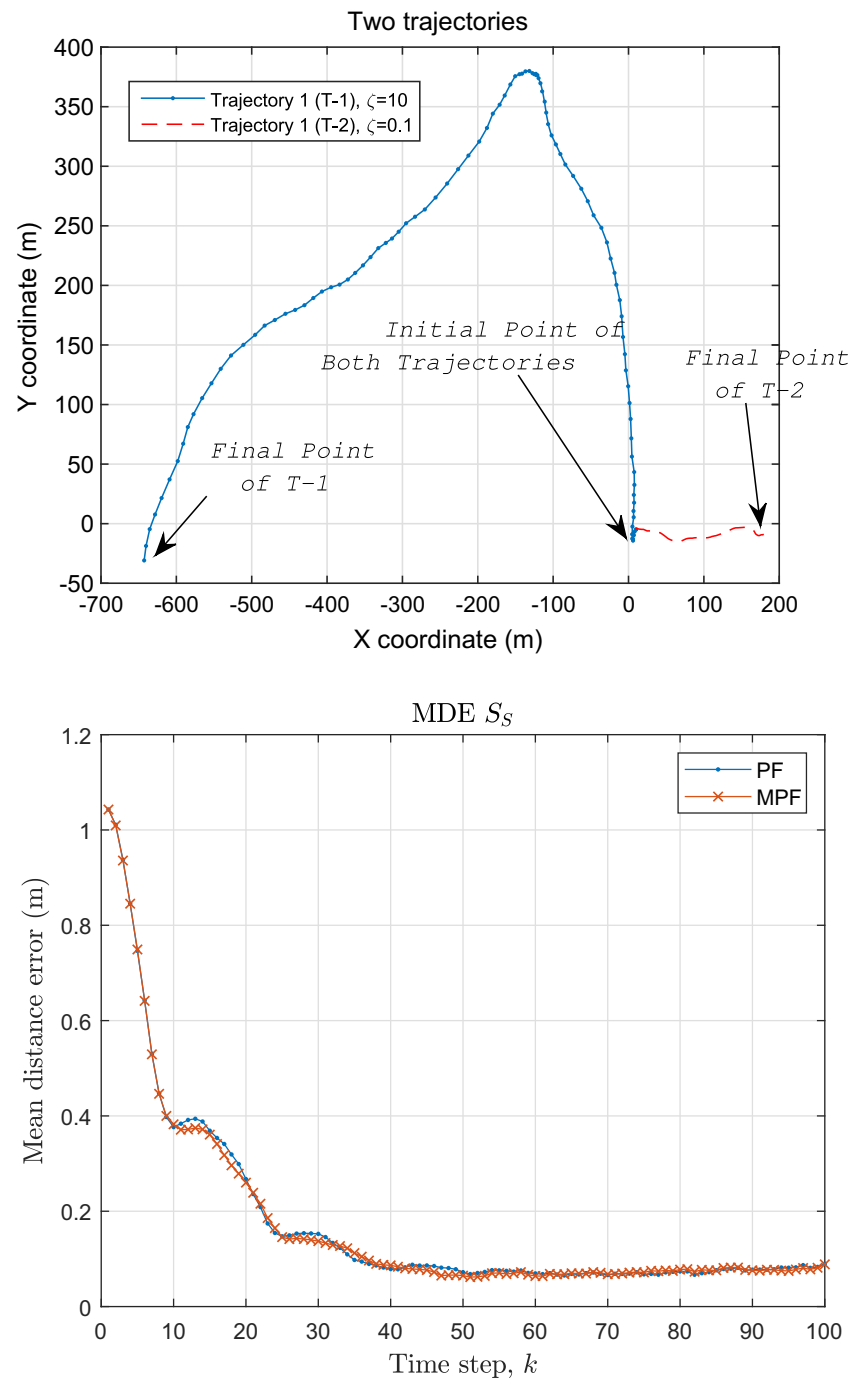

(A)

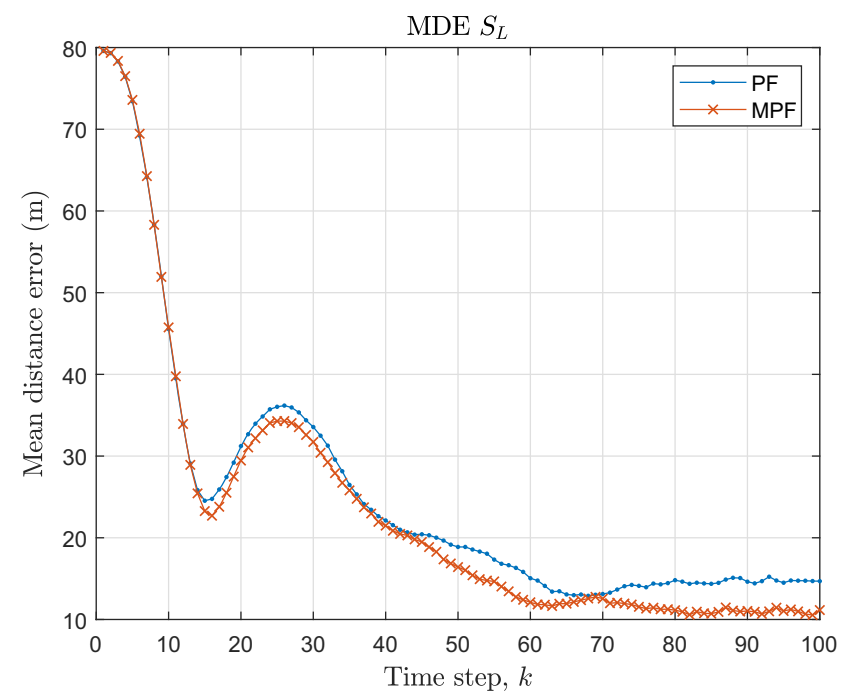

(C)
FIGURE 2 An illustrative example of two trajectories of a target with $\xi=0.1$ and 10 for the state noise during the same elapsed time. It is highly maneuvering with $\xi=10$, ie, $T-1$ [Colour figure can be viewed at wileyonlinelibrary.com]

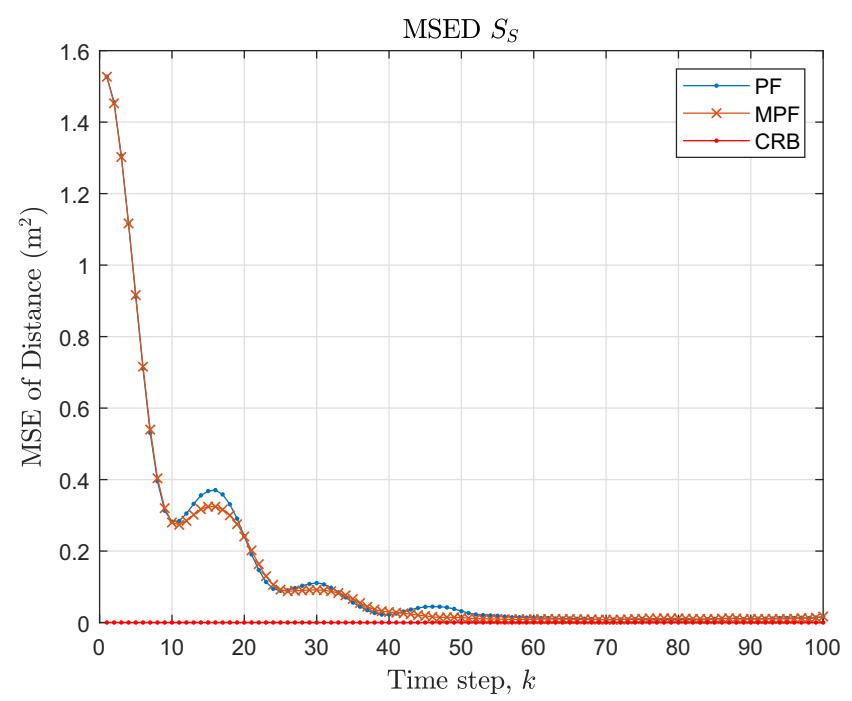

(B)

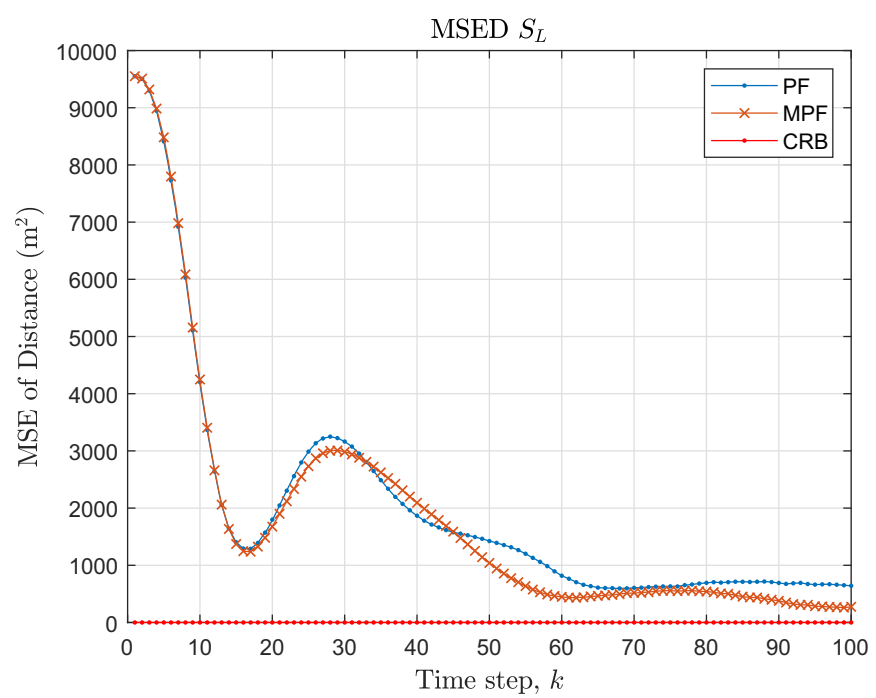

(D)

FIGURE 3 Performance comparison between sequential importance resampling particle filtering (PF) and minimax (PF). Trajectories manifest significant maneuvering when $\xi=1$. Moreover, 500 runs were performed with 500 particles. Comparison with Cramér-Rao lower bound (CRLB) was performed, as derived in the appendix. A, Mean distance error (MDE) with $\xi=0.1$; B, Mean square error of distance (MSED) with $\xi=0.1$;, MDE with $\xi=1$; DSED with $\xi=1$ [Colour figure can be viewed at wileyonlinelibrary.com] 
For the measurement noises, we use $\zeta$ that plays the same role as $\xi$ of the state noise. We have two $\zeta$ s for range and bearing measurements, ie, $\zeta_{R}$ and $\zeta_{B}$, respectively. We select $\xi=10^{-3}, \zeta_{R}=10^{-4}$, and $\zeta_{B}=10^{-5}$ for $S_{S}$ and $\xi=1$, $\zeta_{R}=5 \times 10^{-3}$, and $\zeta_{B}=5 \times 10^{-4}$ for $S_{L}$ The number of total time steps $K=100$. We performed 500 experiments to obtain the mean square error (MSE) of distance (MSED) and mean distance error (MDE) for each location over time. The initial state is generated with a known variance.

The MDE and MSED of SPF, MPF, and Cramér-Rao lower bound (CRLB) are compared in Figure 3. Figures 3A and 3B show the results under $S_{S}(\xi=0.1)$. Figures 3A and 3B show that the performance of SPF and MPF is similar in this low maneuvering scenario while MPF shows marginally better performance than PF. The result with $\xi=1$ is shown in Figures 3C and 3D where we have significantly maneuvering trajectories and MPF shows better performance than PF.

Based on (12), we computed the risk function for both PF frameworks, and the mean values over 500 runs are shown in Figures 4 and 5. Figure 4 shows that the risk by MPF is much higher than that of SPF. For comparison purposes, Figure 5 shows the mean risk of $\mathrm{PF}_{\mathrm{wmax}}$ in addition to those of MPF and SPF. In the $\mathrm{PF}_{\mathrm{wmax}}$ framework, the minimum risk is adopted for each particle as opposed to the case of MPF. The resulting mean risk of $\mathrm{PF}_{\mathrm{wmax}}$ is not bounded, and it diverges significantly such that we cannot perform a minimization to obtain an estimate. Although we did not show results, the estimation performance of $\mathrm{PF}_{\mathrm{wmax}}$ is unduly poor, which is not acceptable. In the following sections, we assess the performance of the minimax versions of PF approaches for other variants such as APF, RPF, and KLDPF.

FIGURE 4 Mean risk over 500 runs with 500 particles in log-scale based on (12). Results regarding only $r_{x}$ are shown, and those of the remaining elements are similar to those of $r_{x}$. Mean risk for sequential importance resampling particle filtering (SIRPF) and minimax particle filtering (MPF) for $\xi=0.1$ and 10. The same values of $\xi_{R}=0.1$ and $x i_{B}=0.001$ are applied for both scenarios [Colour figure can be viewed at wileyonlinelibrary.com]

FIGURE 5 Mean risk over 500 runs with three measurements and 500 particles in log-scale based on (12). Results regarding only $r_{x}$ are shown, and those of the remaining elements are similar to those of $r_{x}$. Mean risk of $\mathrm{PF}_{\mathrm{wmax}}$ is not bounded and diverges over time steps where we use the maximum weight for particles in $\mathrm{PF}_{\mathrm{wmax}}$. MPF, minimax particle filtering; PF, particle filtering; SIRPF, sequential importance resampling particle filtering [Colour figure can be viewed at wileyonlinelibrary.com]
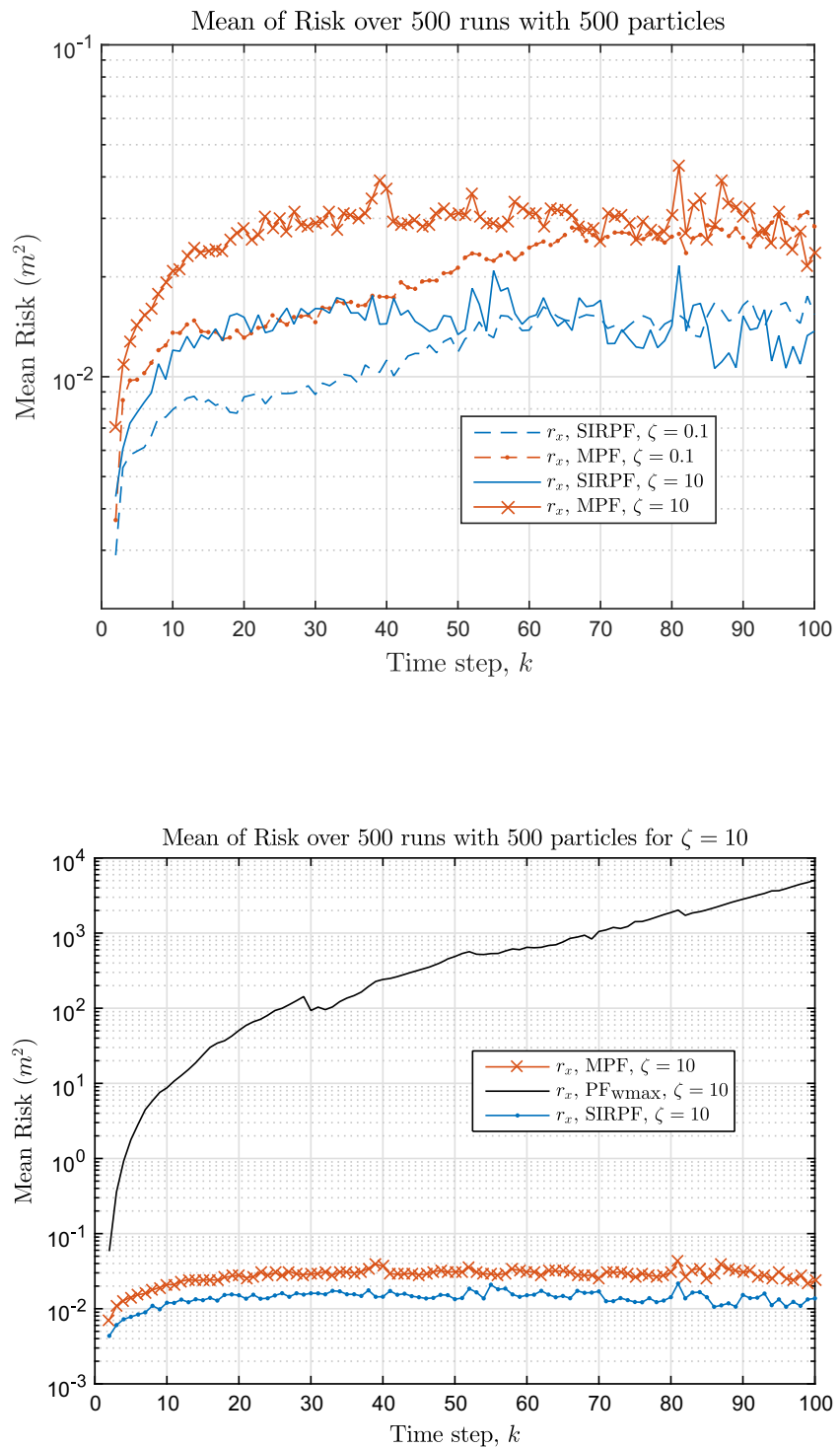


\section{1 | Auxiliary particle filter}

Here, APF was initially introduced in the work of Pitt and Shephard 22 as a variant of SIRPF. We adopted the algorithm based on the work of Arulampalam et al. ${ }^{16}$ In this variant, the algorithm is the same to SIRPF except for the resampling process. Specifically, APF returns to the previous time step after the resampling particles and then propagates the particles again for the next time step. We used $\xi=10^{-3}, \zeta_{R}=10^{-4}$, and $\zeta_{B}=10^{-5}$ for $S_{S}$ and $\xi=10^{-2}, \zeta_{R}=10^{-3}$, and $\zeta_{B}=10^{-4}$ for $S_{L}$ in the experiments.

The results are shown in Figure 6 where minimax-APF (MAPF) outperforms APF even under the scenario of $S_{S}$.

\section{2 | Regularized PF}

In the RPF, PF was modified to resolve the particle impoverishment problem. ${ }^{23}$ Moreover, RPF employs a kernel density that perturbs the state of a particle to achieve the diversity of the particle states. Specifically, the posterior density is

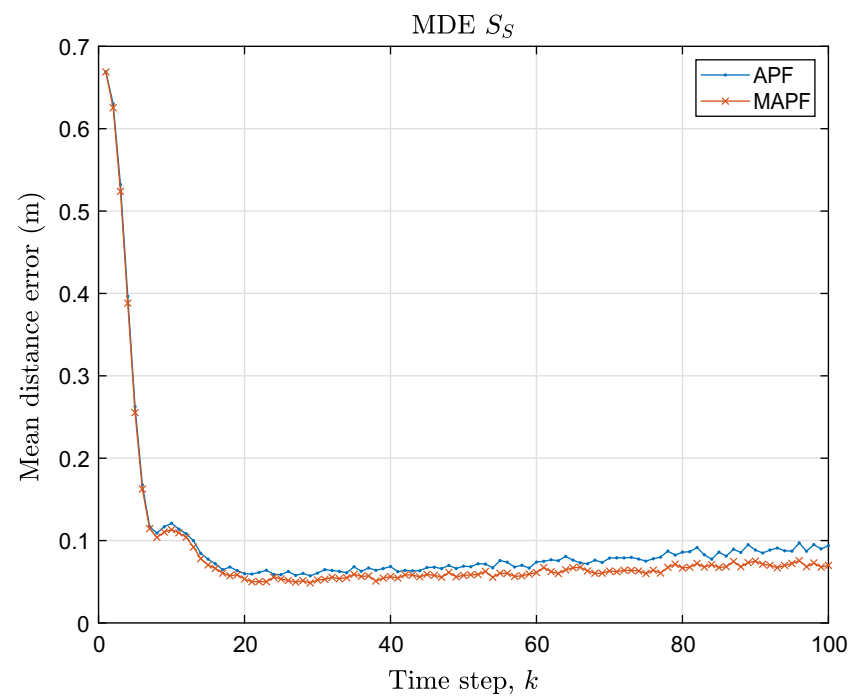

(A)

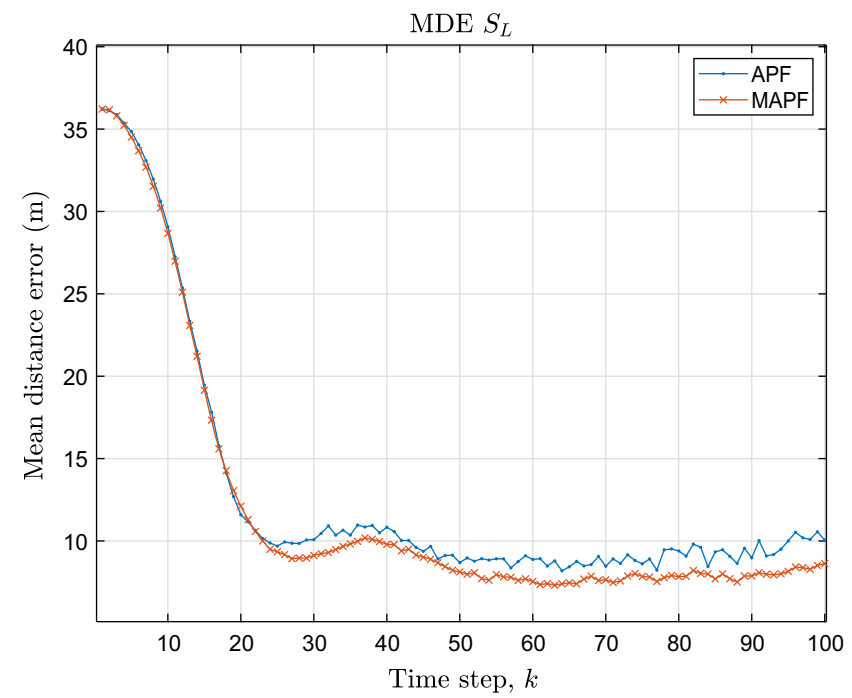

(C)

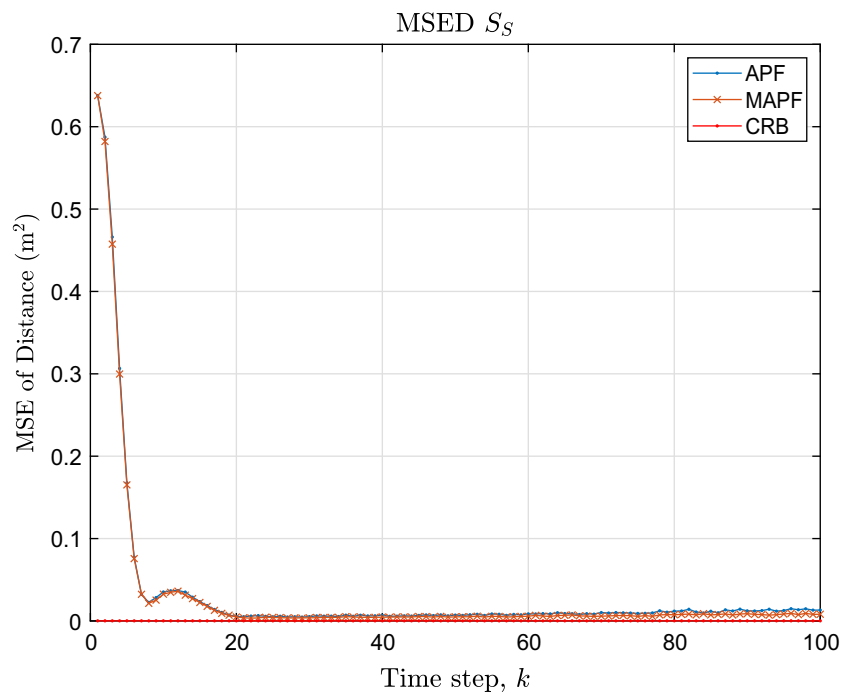

(B)

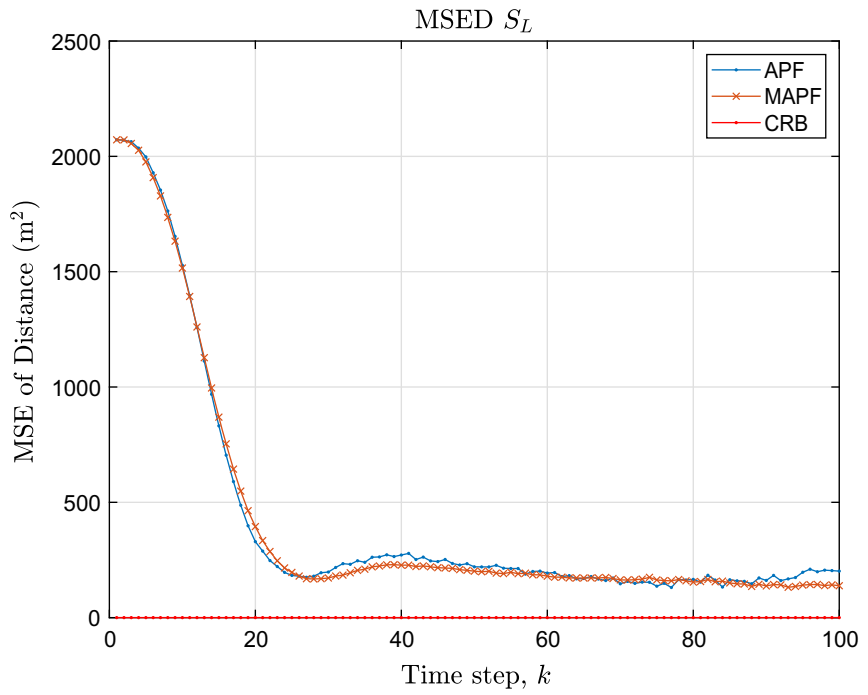

(D)

FIGURE 6 Performance comparison between auxiliary particle filtering (APF) and minimax-APF (MAPF). Here, 500 runs were performed with 500 particles. A, Mean distance error (MDE) with $\xi=10^{-3}$; B, Mean square error of distance (MSED) with $\xi=10^{-3}$; $\mathrm{C}, \mathrm{MDE}$ with $\xi=10^{-2}$; D, MSED with $\xi=10^{-2}$. CRB, Cramér-Rao bound [Colour figure can be viewed at wileyonlinelibrary.com] 
approximated in RPF as follows:

$$
p\left(\boldsymbol{\theta}_{k} \mid z_{1: k}\right) \approx \sum_{i=1}^{M} \omega_{k}^{j_{p}} \Gamma_{\kappa}\left(\boldsymbol{\theta}_{k}-\boldsymbol{\theta}_{k}^{j_{p}}\right),
$$

where $\Gamma_{\kappa}(\theta)=\frac{1}{\kappa^{n_{\theta}}} \Gamma\left(\frac{\theta}{\kappa}\right)$ is the rescaled kernel density for any kernel bandwidth $\kappa>0$ and $n_{\theta}$ is the dimension of the state parameter $\boldsymbol{\theta}$. The optimal choice of the kernel is the Epanechnikov kernel; however, it can be replaced by the Gaussian kernel. ${ }^{24}$ Subsequently, the associated optimal bandwidth is

$$
\kappa_{\mathrm{opt}}=A N^{-\frac{1}{n_{\theta}+4}} \text {, where } A=\left(\frac{4}{n_{\theta}+2}\right)^{\frac{1}{n_{\theta}+4}} .
$$

In this study, we use $n_{\theta}=4$ with $N=500$ particles to perform experiments and compare the performances of RPF and minimax-RPF (MRPF). We used $\xi=10^{-3}, \zeta_{R}=10^{-4}$, and $\zeta_{B}=10^{-5}$ for $S_{S}$ and $\xi=1, \zeta_{R}=0.1$, and $\zeta_{B}=10^{-2}$ for $S_{L}$ in the experiments. The results are shown in Figure 7, where MRPF outperforms RPF even under the scenario of $S_{S}$, as shown in Figures 7A and 7B. Under both scenarios, MRPF outperforms RPF.

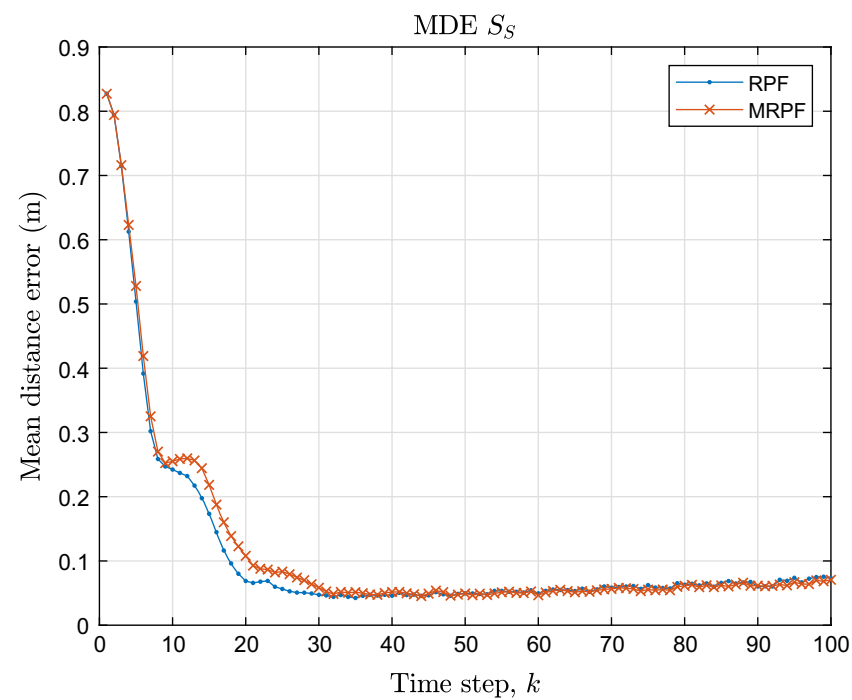

(A)

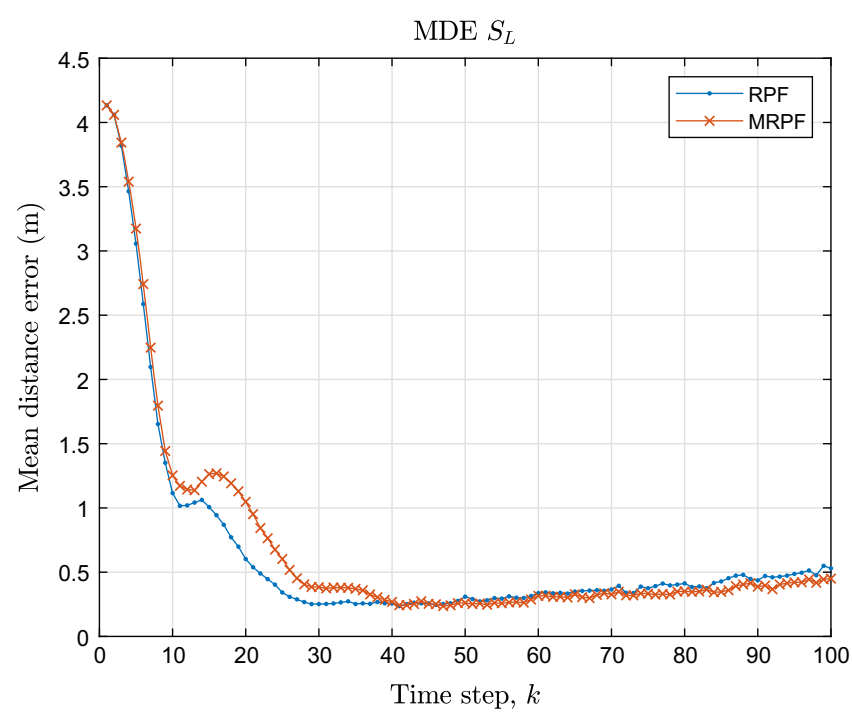

(C)

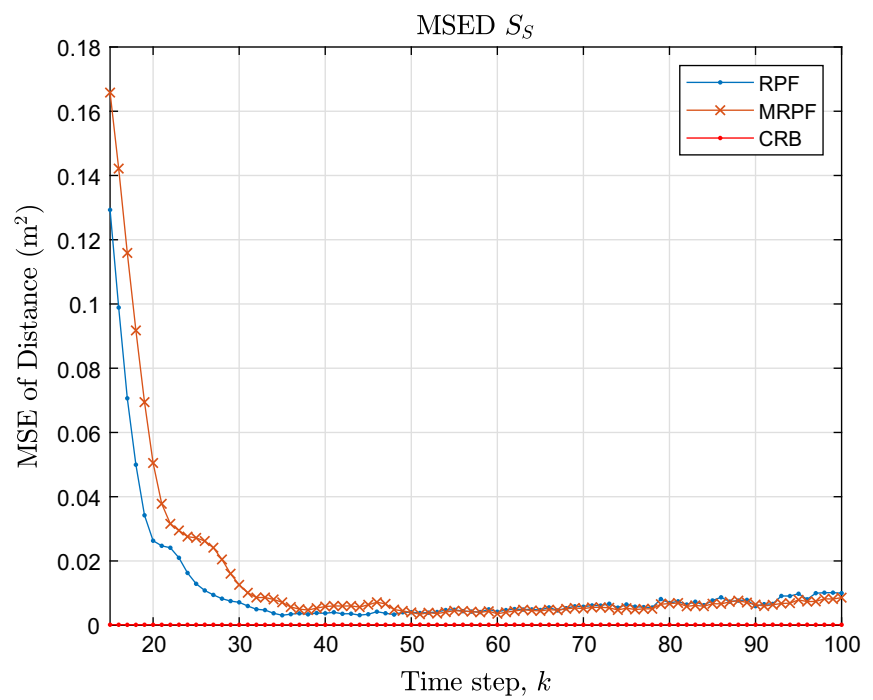

(B)

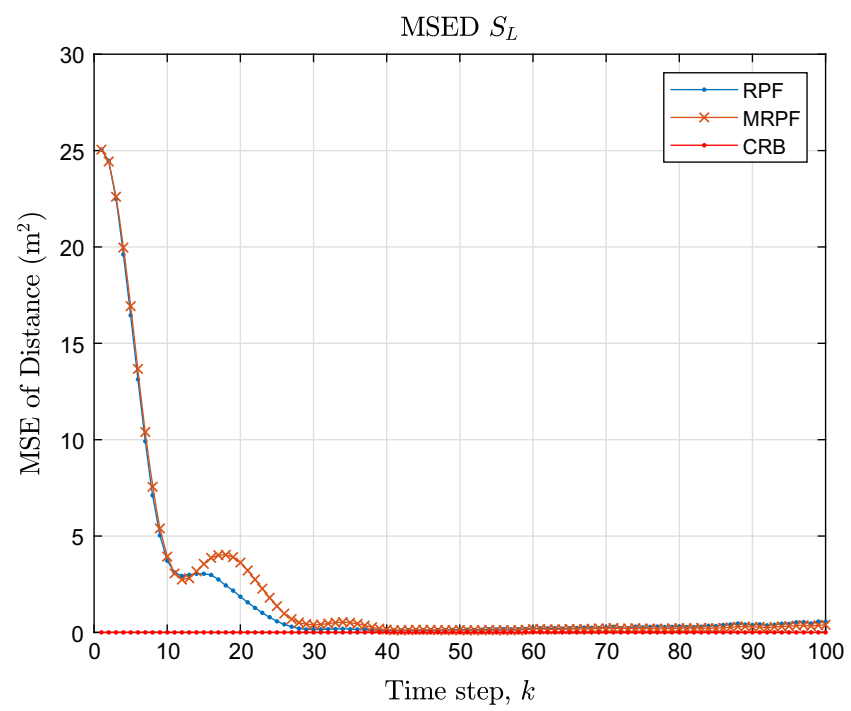

(D)

FIGURE 7 Performance comparison between regularized particle filtering (RPF) and minimax-RPF (MRPF). Here, 500 runs were performed with 500 particles. A, Mean distance error (MDE) with $\xi=0.1$; , Mean square error of distance (MSED) with $\xi=0.1$;, MDE with $\xi=1$; D, MSED with $\xi=1$. CRB, Cramér-Rao bound [Colour figure can be viewed at wileyonlinelibrary.com] 


\section{3 | Kullback-Leibler divergence PF}

A primary feature of KLDPF is that the number of employed particles is optimized adaptively based on a predefined error bound at every time step. ${ }^{8}$ While it may not show better performance than SPF, the number of employed particles is dynamically adjusted to reduce redundant particles and also unnecessary computations accordingly. We adopt the KLDPF algorithm introduced in the work of $\mathrm{Li}$ et $\mathrm{al}^{9}$ with an error bound of 0.01 . The initial number of employed particles is 250 , and the maximum number of particles is bounded by 500. The probability bound is 0.01 ; the bin size is $1 / 2 \times \sqrt{\delta_{u_{k}}} \times 4$ as suggested in the aforementioned work, ${ }^{9}$ where $1 / 2$ is from $A 2 ; \delta_{\boldsymbol{u}_{k}}$ is the variance of the state noise with respect to $v_{x}$ or $v_{y}$. Although KLDPF adaptively optimizes the number of particles at every time step, the algorithm inherently requires a higher computational cost than those of other PF variants.

We used $\xi=10^{-3}, \zeta_{R}=10^{-4}$, and $\zeta_{B}=10^{-5}$ for $S_{S}$ and $\xi=10, \zeta_{R}=0.1$, and $\zeta_{B}=10^{-3}$ for $S_{L}$ in the experiments. Minimax-KLDPF (MKLDPF) outperforms KLDPF in both scenarios as shown in Figure 8. Figure 9 shows the mean number of the employed particles for the scenarios of highly maneuvering target tracking, where MKLDPF requires marginally more number of particles than that required by regular KLDPF.

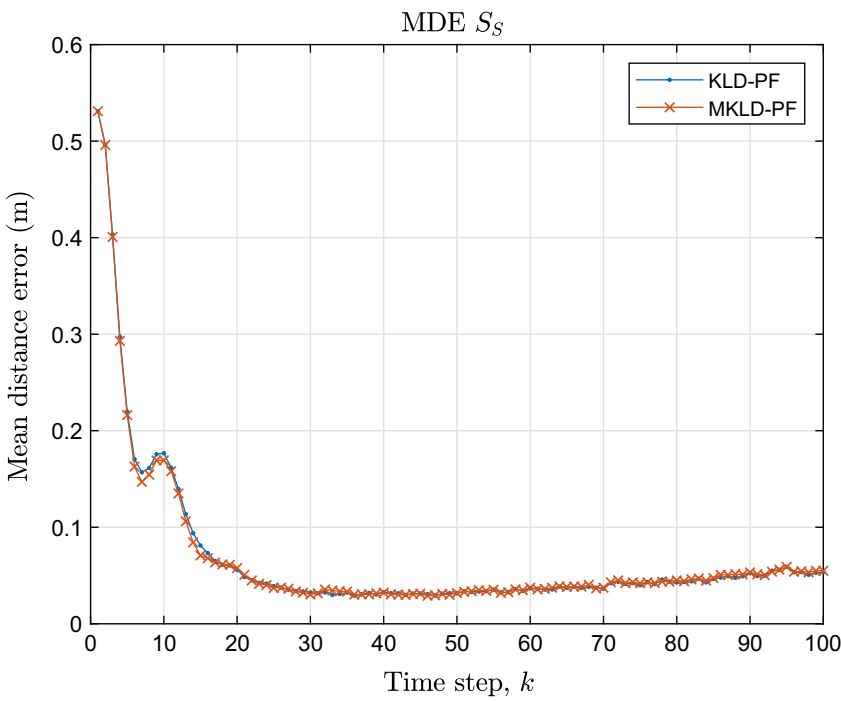

(A)

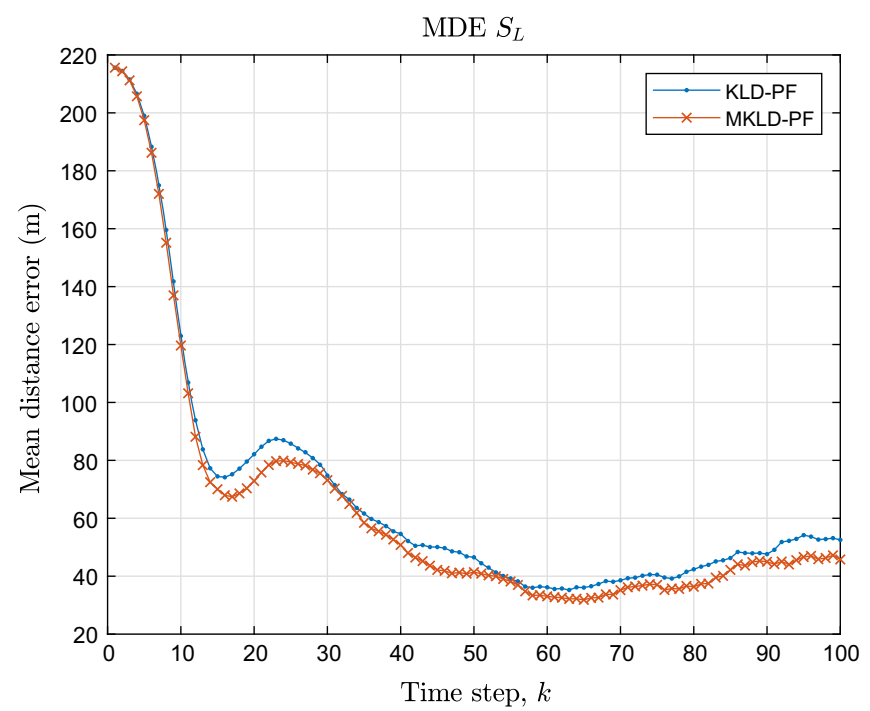

(C)

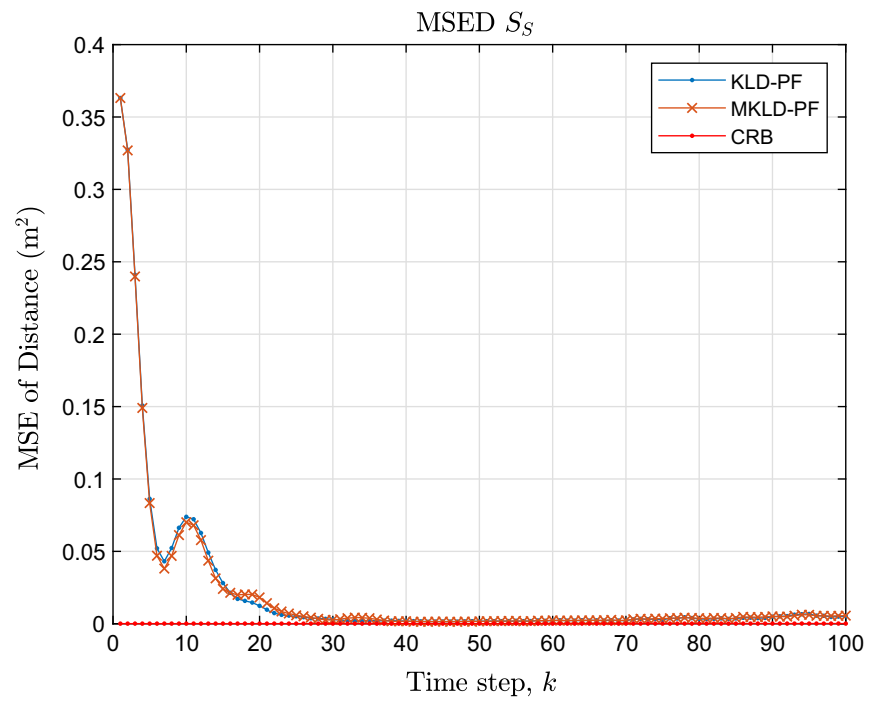

(B)

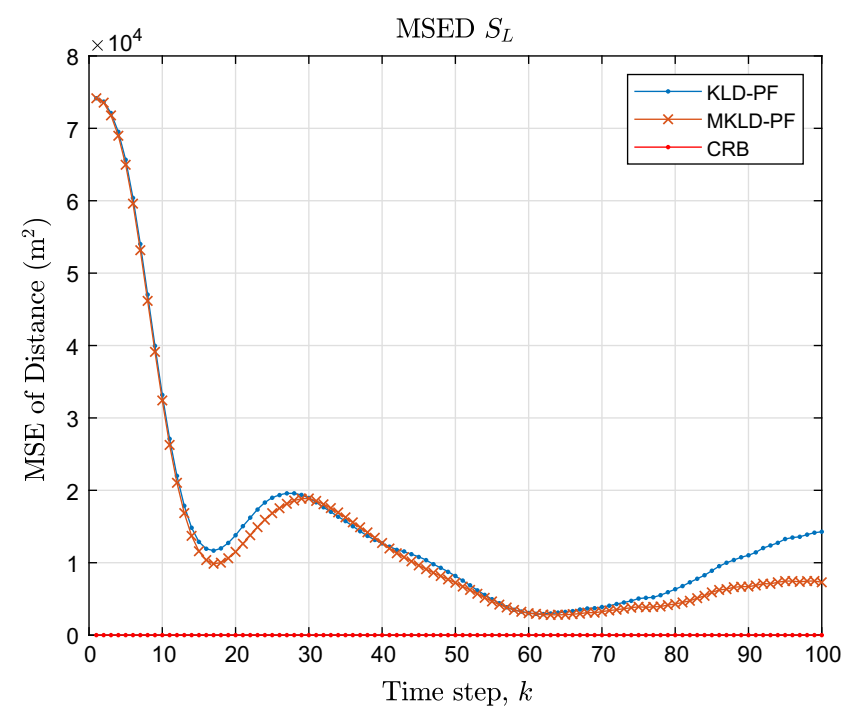

(D)

FIGURE 8 Performance comparison between Kullback-Leibler divergence particle filtering (KLDPF) and minimax-KLDPF (MKLDPF). The initial number of particles is 200 with a maximum of 500 particles. A, Mean distance error (MDE) with $\xi=10^{-3}$; B, Mean square error of distance (MSED) with $\xi=10^{-3}$; C, MDE with $\xi=10^{-2}$; D, MSED with $\xi=10^{-2}$. CRB, Cramér-Rao bound [Colour figure can be viewed at wileyonlinelibrary.com] 
FIGURE 9 The number of employed particles with 500 maximum particles in Kullback-Leibler divergence particle filtering (KLDPF), two measurements, and $\xi=10$. MKLDPF, minimax-KLDPF [Colour figure can be viewed at wileyonlinelibrary.com]

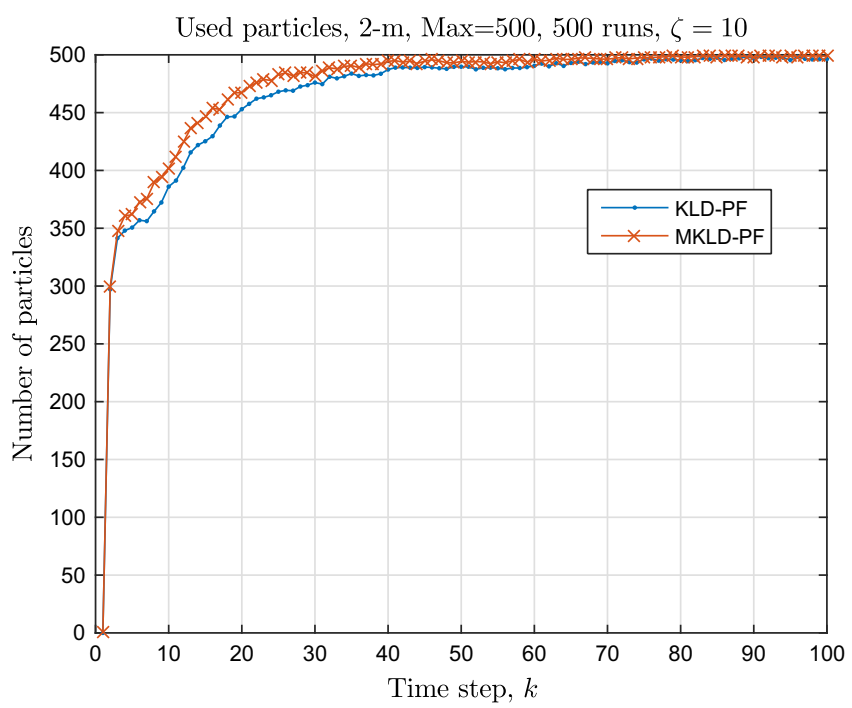

\subsection{Comparison with interactive multiple model filters}

Finally, we adopt the minimax strategy to the IMM particle filtering (IMM-PF). A conventional IMM-EKF evaluation is compared to the IMM-minimax-PF (IMM-MPF) approach in this experiment. We refer to the works of $\mathrm{Xu}^{25}$ and $\mathrm{Li}$ and Wang ${ }^{3}$ for IMM-EKF and IMM-PF, respectively. The results are shown in Figure 10 where IMM-MPF outperforms IMM-PF and IMM-EKF. We used $\xi=0.1, \zeta_{R}=10^{-2}$, and $\zeta_{B}=10^{-2}$ in the experiments.

\section{5 | Discussion}

The proposed MPF was derived based on the same criterion as a Bayesian method that minimizes MSE, except for the main difference that the proposed approach adopts the maximum risk among various options of risk functions. Unlike conventional Bayesian-MMSE-PF approaches where the weight of each particle is computed based on joint probability density (ie, usually multiplication of likelihood functions of all measurements assuming white Gaussian noise), the proposed minimax approach selects the minimum weight based on a single measurement for each particle. We obtain the estimate based on these weights that are selected to minimize the maximized risk. This strategy enables the significantly reduced variance of the weights of particles. We can obtain improved quality of particles by the reduced variance and eventually improve the tracking performance. Therefore, the proposed minimax strategy makes the filter robust against the degeneracy problem of standard PF approaches.

FIGURE 10 Mean distance error comparison of interactive multiple model extended Kalman filter (IMM-EKF), IMM particle filtering (IMM-PF), and IMM minimax-PF (IMM-MPF) under $S_{S}$, where $\sigma_{u_{\omega}}$ is the noise variance of the turn rate. MDE, mean distance error [Colour figure can be viewed at wileyonlinelibrary.com]

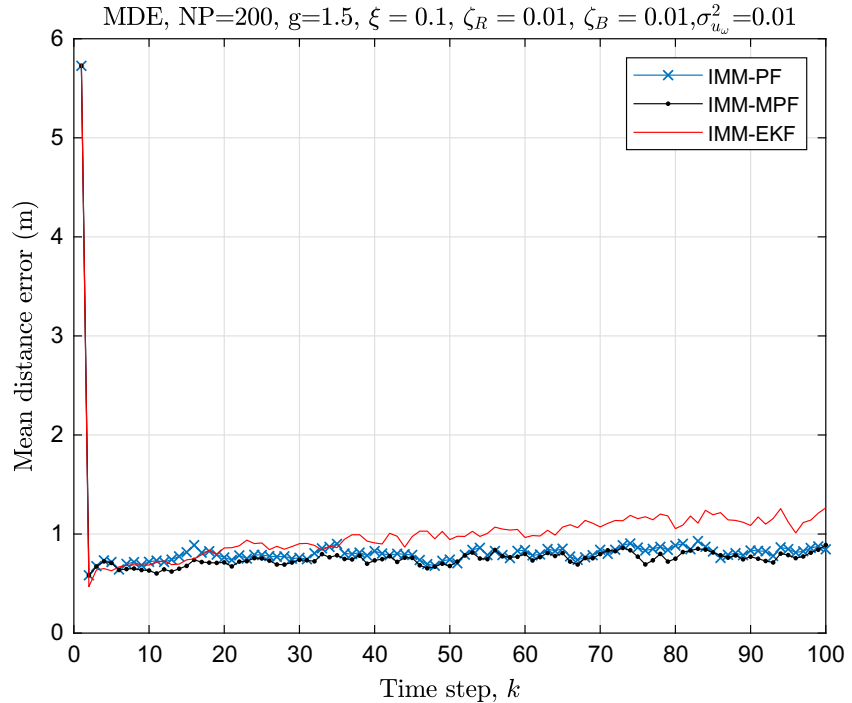


The proposed approach is computationally efficient because it does not require computing a joint probability for the weight of a particle. Computation of joint probability based on multiple measurements typically undergoes substantial computational load owing to additional multiplications.

We compared the performances of all minimax-PF approaches together. The results are shown in Figures 11 and 12 under $S_{S}$ and $S_{L}$ scenarios, respectively. We used $\xi=10^{-3}, \zeta_{R}=10^{-4}$, and $\zeta_{B}=10^{-5}$ for $S_{S}$ and $\xi=1, \zeta_{R}=0.1$, and $\zeta_{B}=10^{-2}$ for $S_{L}$ in the experiments. All minimax PFs show similar performance under the scenario of $S_{S}$, while both MAPF and MRPF show similarly better performance compared with the other two approaches under the scenario of $S_{L}$.

We also computed the mean variance of the weights of particles during the previous experiments for all minimax-PFs. Figure 13 shows the mean variances of each minimax-PF under both scenarios of $S_{S}$ and $S_{L}$. The results show that we can obtain significantly reduced variances by minimax-PFs compared with regular PFs regardless of the scenarios.

We obtained normalized estimation error squared (NEES) and average NEES (ANEES) for all particle filtering approaches concerning both regular and minimax versions. We used $\xi=10^{-3}, \zeta_{R}=10^{-4}$, and $\zeta_{B}=10^{-5}$, which is close to the scenario of $S_{S}$ in the experiments. Based on the work of Li et al, ${ }^{26}$ NEES and ANEES are defined, respectively, as follows:

$$
\begin{aligned}
& \chi_{r}=\left(\boldsymbol{\theta}_{r}-\hat{\boldsymbol{\theta}}_{r}\right)^{\top} P_{r}^{-1}\left(\boldsymbol{\theta}_{r}-\hat{\boldsymbol{\theta}}_{r}\right) \\
& \bar{\chi}_{r}=\frac{1}{L R} \sum_{r=1}^{R} \chi_{r},
\end{aligned}
$$
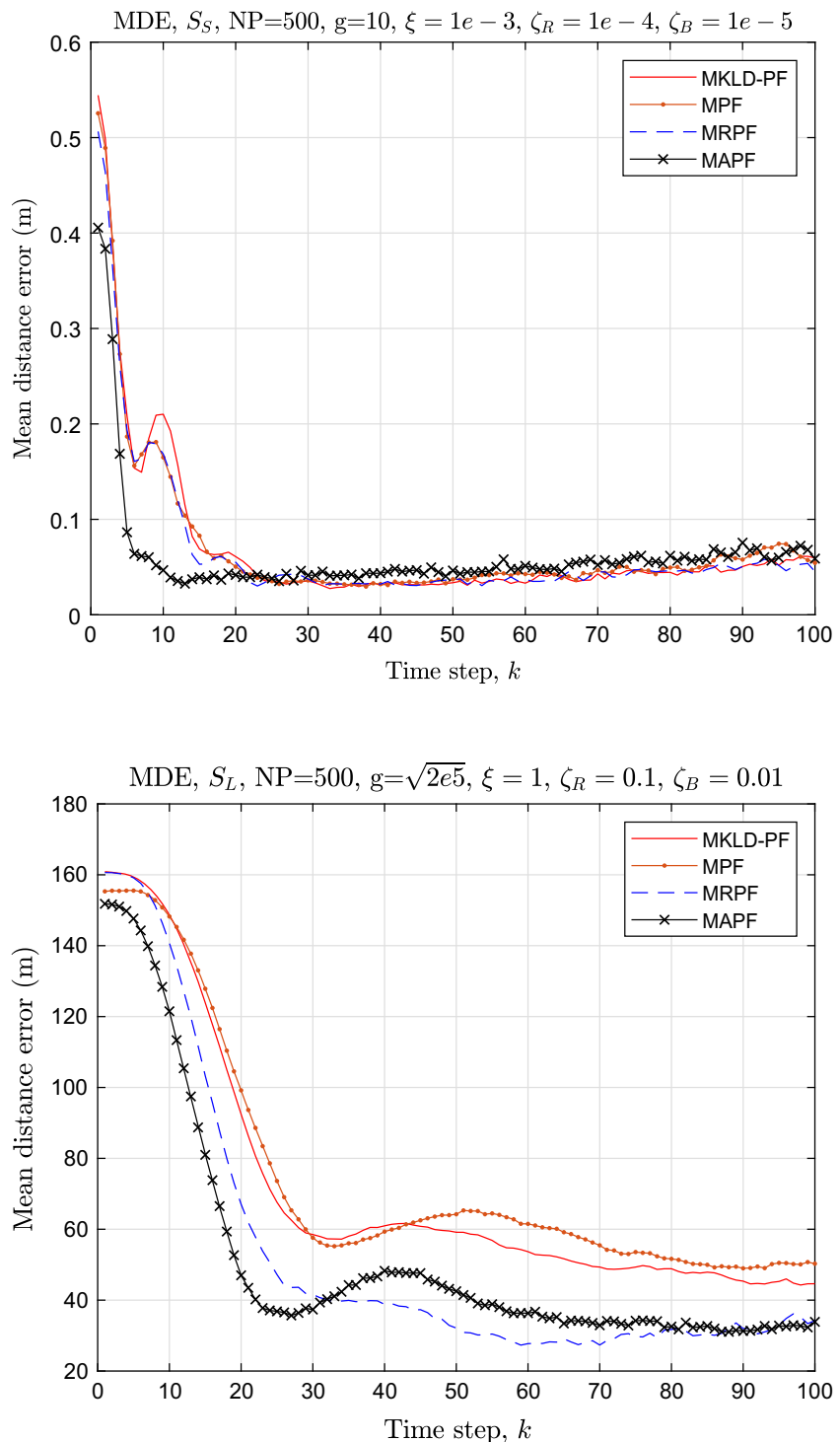

FIGURE 11 Results of mean distance error (MDE) for minimax approaches under $S_{S}$. All show similar performance. MAPF, minimax auxiliary particle filtering; MKLDPF, Kullback-Leibler divergence particle filtering; MPF, minimax particle filtering; MRPF, minimax regularized particle filtering [Colour figure can be viewed at wileyonlinelibrary.com]

FIGURE 12 Results of mean distance error (MDE) for minimax particle filtering (MPF) approaches under $S_{L}$. Minimax auxiliary particle filtering (MAPF) and minimax regularized particle filtering (MRPF) show similarly better performance than the other two. MKLDPF, minimax Kullback-Leibler divergence particle filtering [Colour figure can be viewed at wileyonlinelibrary.com] 


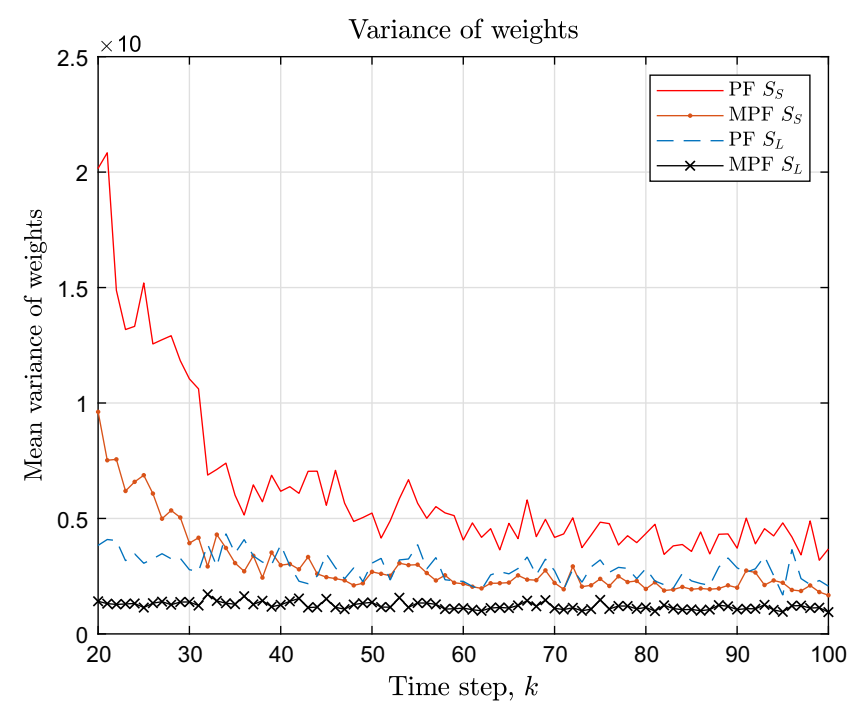

(A)

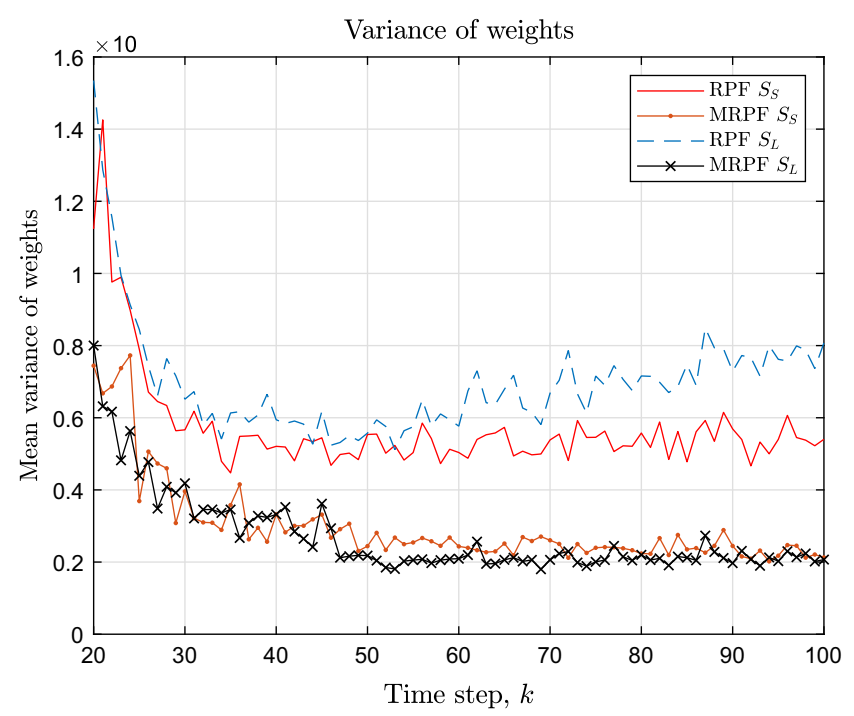

(C)

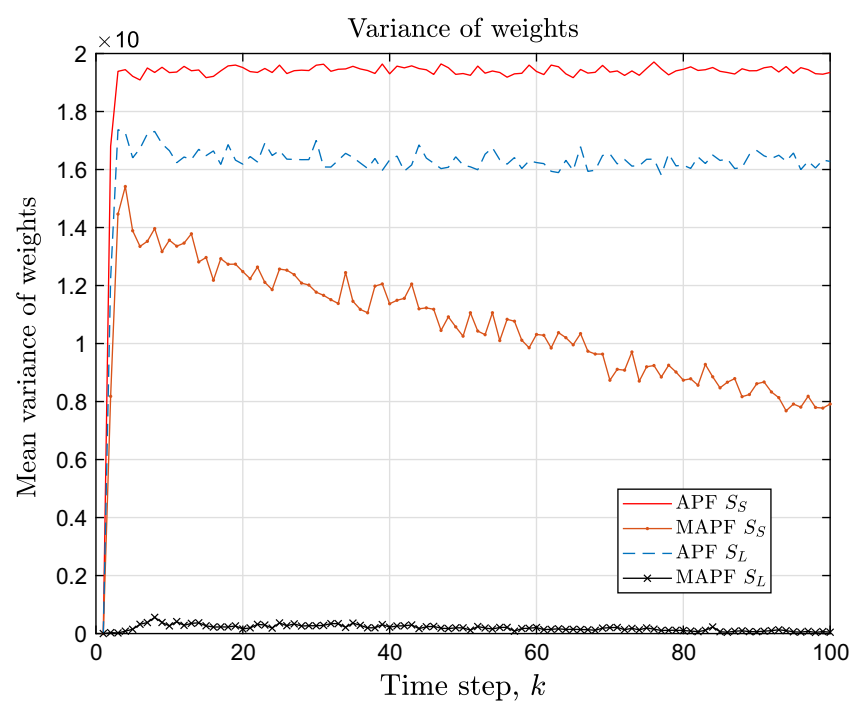

(B)

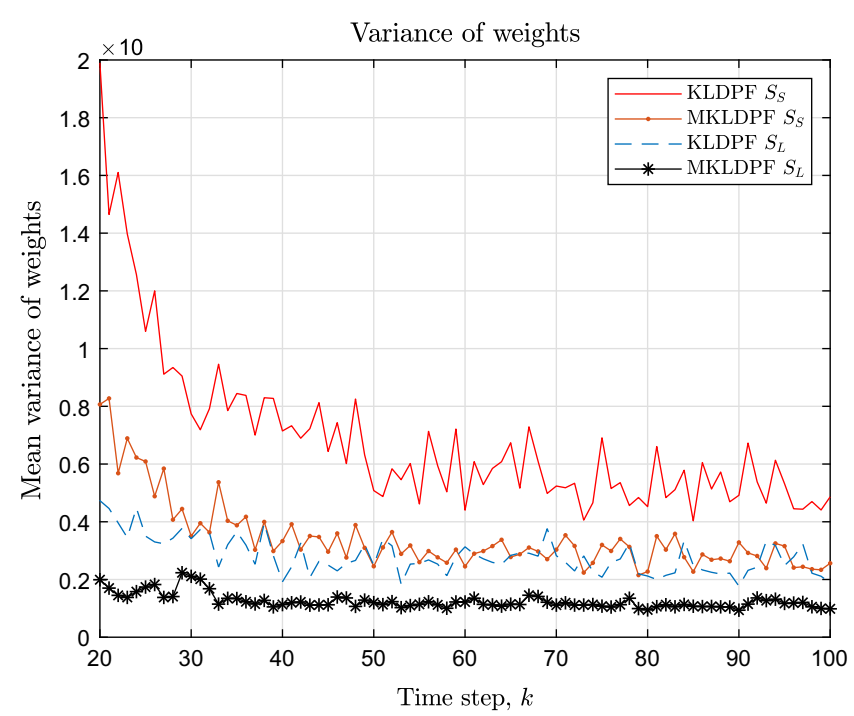

(D)

FIGURE 13 Mean variance of the weights of particles. The results are shown from at the time step 20 for particle filtering (PF), regularized PF (RPF), and Kullback-Leibler divergence PF (KLDPF) for clear visibility. A, Mean variance: PF and minimax-PF (MPF); B, Mean variance: auxiliary PF (APF) and minimax-APF (MAPF); C, Mean variance: RPF and minimax-RPF (MRPF); D, Mean variance: KLDPF and minimax-KLDPF (MKLDPF) [Colour figure can be viewed at wileyonlinelibrary.com]

where $r$ is the index of a run, $L$ is the dimension of the state $\theta, P$ is the estimator-provided error covariance, and $R$ is the number of runs, respectively. According to the work of Li et al, ${ }^{26}$ ANEES is recommended for testing whether an estimator should be rejected as not credible or is optimistic or pessimistic. The closer to 1 the ANEES is, the more credible the estimator. If ANEES is much greater than 1, the actual estimation error is much larger than what the estimator believes (ie, the estimator is unduly optimistic); if ANEES is much smaller than 1, the actual estimation error is much smaller than what the estimator believes (ie, the estimator is unduly pessimistic). Figure 14 shows the result of ANEES for four of all particle filtering methods concerning both regular and minimax versions. The results are under $S_{S}$ and various sizes of variances of initial values. The ANEES depends on the noises and the magnitude of the variance of the initial values; however, most results show more or less around 1 with small noises that we applied in these 300 runs. In our case, $L=4$. Most of the approaches are somewhat too pessimistic, ie, the actual estimation error is much smaller than what the estimator believes. 


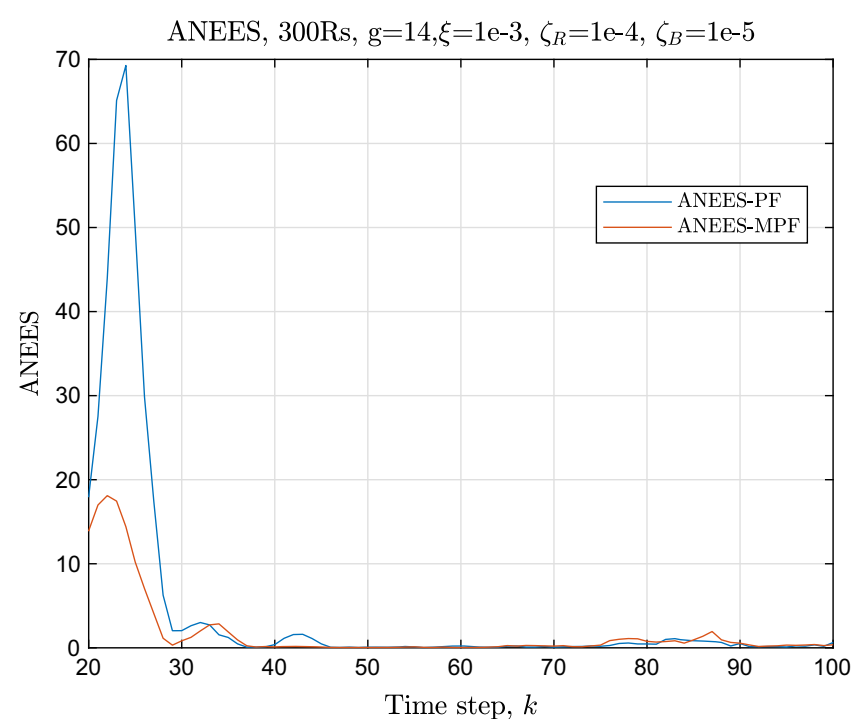

(A)

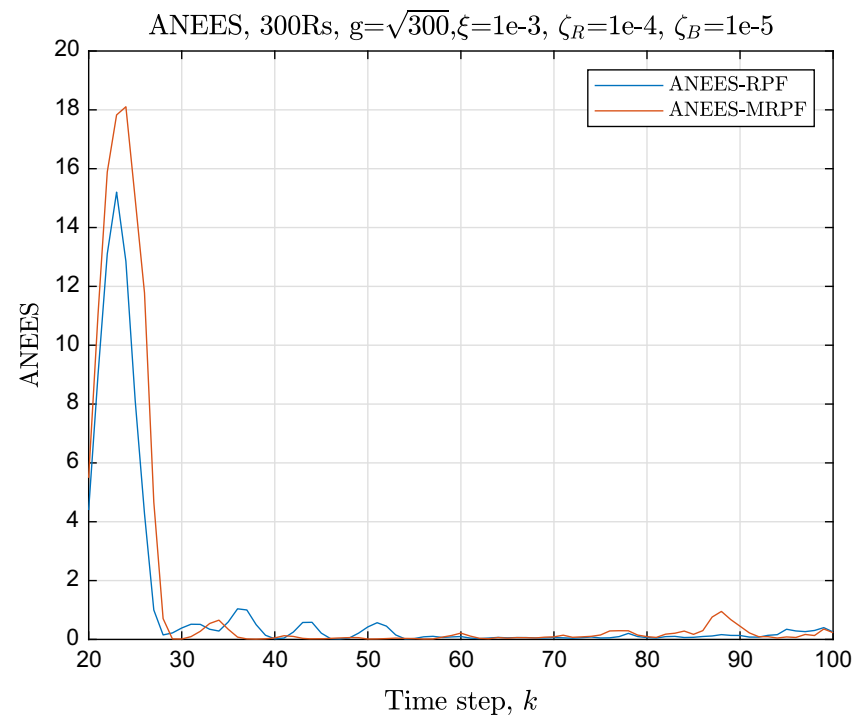

(C)

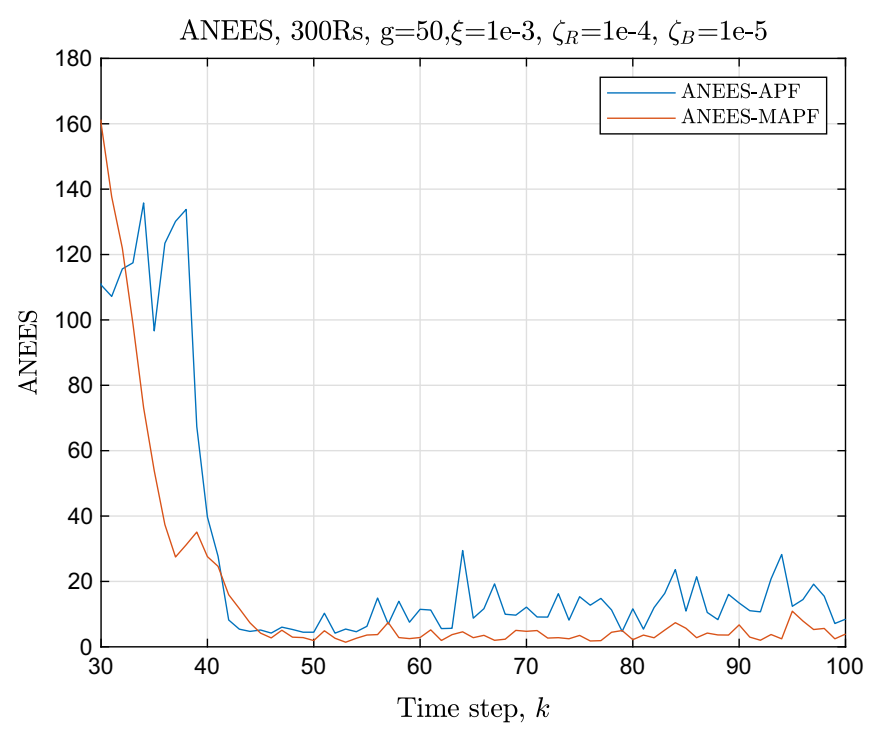

(B)

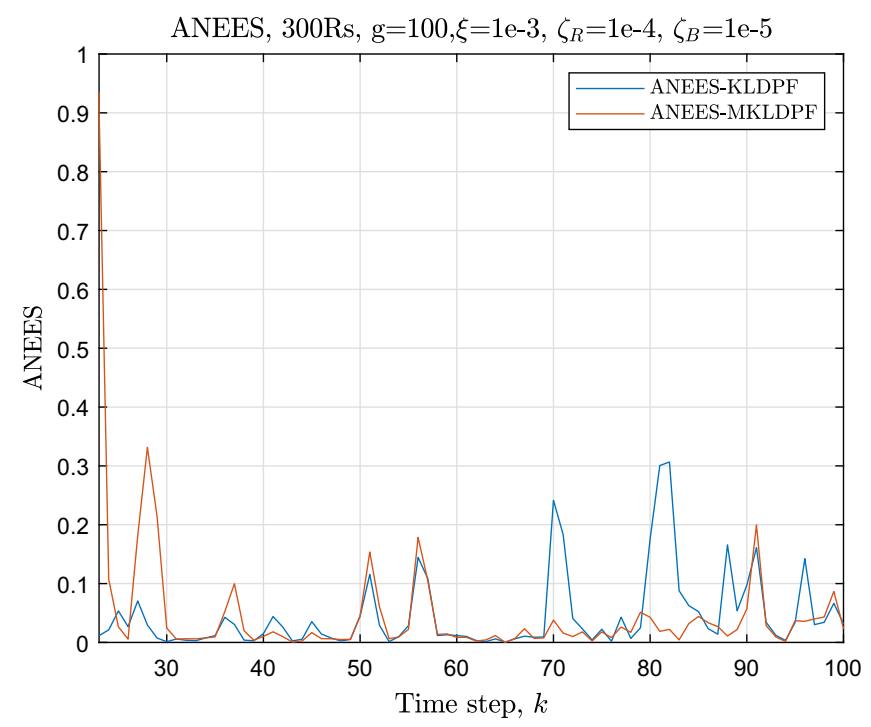

(D)

FIGURE 14 Average normalized estimation error squared (ANEES) with the scenario of $S_{S}$. A, ANEES for particle filtering (PF) vs minimax-PF (MPF); B, ANEES for auxiliary PF (APF) vs minimax-APF (MAPF); C, ANEES for regularized PF (RPF) vs minimax-RPF (MRPF); D, ANEES for Kullback-Leibler divergence PF (KLDPF) vs minimax-KLDPF (MKLDMPF) [Colour figure can be viewed at wileyonlinelibrary.com]

\section{5 | CONCLUSION}

In this paper, we proposed a new PF framework for highly maneuvering target tracking. The minimax strategy was adopted in this framework, which results in the significantly reduced variance of the weights of particles and in the robustness against the degeneracy problem of regular PF approaches. The robustness made it possible to overcome the difficulty in tracking a highly maneuvering target where particularly the state noise variance is large. We verified the effectiveness of the proposed MPF by experiments in various scenarios and showed that MPF outperforms nonminimax PF approaches. The proposed minimax strategy can be adopted for any other variants of PF provided that multiple measurements are available, including sensor networks environment. The computational complexity in MPF was reduced because the computation of complex joint probability density function was avoided in the proposed algorithm. We further showed that the minimax strategy is effective in the form of IMM-PF and minimax IMM-PF outperformed IMM-PF and conventional IMM-EKF. 


\section{ACKNOWLEDGEMENTS}

This work was supported by the Basic Science Research Program through the National Research Foundation of Korea funded by the Ministry of Education under grants NRF-2016R1D1A1A09918304 and NRF-2019R1I1A1A01058976.

\section{CONFLICT OF INTEREST}

The authors declare no potential conflict of interests.

\section{ORCID}

Hyung-Min Park (iD) https://orcid.org/0000-0002-7105-5493

\section{REFERENCES}

1. Bekir E. Adaptive Kalman filter for tracking maneuvering targets. J Guid Control Dyn. 1983;6(5):414-416.

2. Boers Y, Driessen JN. Interacting multiple model particle filter. IEE Proceedings Radar Sonar Navigation. 2003;150(5):344-349.

3. Li H-W, Wang J. Particle filter for manoeuvring target tracking via passive radar measurements with glint noise. IET Radar Sonar Navig. 2012;6(3):180-189.

4. Zhang T, Liu S, Xu C, Liu B, Yang M-H. Correlation particle filter for visual tracking. IEEE Trans Image Process. 2018;27(6):2676-2687.

5. Closas P, Guillamon A. Sequential estimation of intrinsic activity and synaptic input in single neurons by particle filtering with optimal importance density. EURASIP J Adv Signal Process. 2017;2017(1):65.

6. Guarniero P, Johansen AM, Lee A. The iterated auxiliary particle filter. J Am Stat Assoc. 2017:1-12.

7. Murangira A, Musso C, Dahia K. A mixture regularized rao-blackwellized particle filter for terrain positioning. IEEE Trans Aerosp Electron Syst. 2016;52(4):1967-1985.

8. Fox D. Adapting the sample size in particle filters through KLD-sampling. Int J Robot Res. 2003;22(12):985-1003.

9. Li T, Sun S, Sattar TP. Adapting sample size in particle filters through KLD-resampling. Electron Lett. 2013;49(12):740-742.

10. Zhou T, Peng D, Xu C, Zhang W, Shen J. Adaptive particle filter based on Kullback-Leibler distance for underwater terrain aided navigation with multi-beam sonar. IET Radar Sonar Navig. 2018;12(4):433-441.

11. Kotecha JH, Djuric PM. Gaussian sum particle filtering. IEEE Trans Signal Process. 2003;51(10):2592-2601.

12. Lim J, Hong D. Gaussian particle filtering approach for carrier frequency offset estimation in OFDM systems. IEEE Signal Process Lett. 2013;20(4):367-370.

13. Míguez J, Bugallo MF, Djurić PM. A new class of particle filters for random dynamic systems with unknown statistics. EURASIP J Adv Signal Process. 2004;2004(15):1-17.

14. Lim J, Hong D. Cost reference particle filtering approach to high-bandwidth tilt estimation. IEEE Trans Ind Electron. 2010;57(11):3830-3839.

15. Lim J. Particle filtering for nonlinear dynamic state systems with unknown noise statistics. Nonlinear Dynamics. 2014;78(2):1369-1388.

16. Arulampalam MS, Maskell S, Gordon N, Clapp T. A tutorial on particle filters for online nonlinear/non-Gaussian Bayesian tracking. IEEE Trans Signal Process. 2002;50(2).

17. Gustafsson F, Gunnarsson F, Bergman N, Forssell U, Jansson J, Karlsson R, Nordlund P-J. Particle filters for positioning, navigation, and tracking. IEEE Trans Signal Process. 2002;50(2):425-437.

18. Djuric PM, Vemula M, Bugallo MF. Target tracking by particle filtering in binary sensor networks. IEEE Trans Signal Process. 2008;56(6):2229-2238.

19. Lim J. A target tracking based on bearing and range measurement with unknown noise statistics. J Electr Eng Technol. 2013;8(6):1520-1529.

20. Shen X-M, Deng L. Game theory approach to discrete H/sub/spl infin//filter design. IEEE Trans Signal Process. 1997;45(4):1092-1095.

21. Simon D. A game theory approach to constrained minimax state estimation. IEEE Trans Signal Process. 2006;54(2):405-412.

22. Pitt MK, Shephard N. Filtering via simulation: auxiliary particle filters. J Am Stat Assoc. 1999;94(446):590-599.

23. Lim J. Performance degradation due to particle impoverishment in particle filtering. J Electr Eng Technol. 2014;9(6):2107-2113.

24. Musso C, Oudjane N, Le Gland F. Improving regularised particle filters. In: Sequential Monte Carlo Methods in Practice. New York, NY: Springer; 2001:247-271.

25. Xu S. Particle Filtering for Systems with Unknown Noise Probability Distributions [PhD Thesis]. Stony Brook, NY: Department of Electrical and Computer Engineering, Stony Brook University-SUNY; 2006.

26. Li XR, Zhao Z, Jilkov VP. Practical measures and test for credibility of an estimator. In: Proceedings of the Workshop on Estimation, Tracking and Fusion: A Tribute to Yaakov Bar-Shalom; 2001; Monterey, CA.

27. Kay SM. Fundamentals of Statistical Signal Processing. Vol. 1. Upper Saddle River, NJ: Prentice Hall; 1993.

How to cite this article: Lim J, Kim H-S, Park H-M. Minimax particle filtering for tracking a highly maneuvering target. Int J Robust Nonlinear Control. 2020;30:636-651. https://doi.org/10.1002/rnc.4785 


\section{APPENDIX}

The variance of any unbiased estimate, $\hat{\boldsymbol{\theta}}$ is bounded as follows:

$$
\operatorname{Var}\left(\hat{\boldsymbol{\theta}}_{t}\right) \geq\left[\mathbf{I}^{-1}(\boldsymbol{\theta})\right]_{t t}
$$

where $\mathbf{I}$ is the Fisher information matrix, $\theta$ is a vector state, and $t$ is the element index. When the measurement noise is Gaussian and the measurement is given by

$$
\boldsymbol{z} \sim \mathcal{N}\left(f(\boldsymbol{\theta}), \boldsymbol{C}_{\boldsymbol{v}}(\boldsymbol{\theta})\right)
$$

we can obtain

$$
[\mathbf{I}(\theta)]_{t l}=\left[\frac{\partial f(\boldsymbol{\theta})}{\partial \theta_{t}}\right]^{\top} \boldsymbol{C}_{\boldsymbol{v}}^{-1}(\boldsymbol{\theta})\left[\frac{\partial f(\boldsymbol{\theta})}{\partial \theta_{l}}\right]+\frac{1}{2} \operatorname{tr}\left[\left(\boldsymbol{C}_{\boldsymbol{v}}{ }^{-1}(\boldsymbol{\theta}) \frac{\partial \boldsymbol{C}_{\boldsymbol{v}}(\boldsymbol{\theta})}{\partial \theta_{t}} \boldsymbol{C}_{\boldsymbol{v}}{ }^{-1}(\boldsymbol{\theta}) \frac{\partial \boldsymbol{C}_{\boldsymbol{v}}(\boldsymbol{\theta})}{\partial \theta_{l}}\right)\right] .
$$

For three measurements, $f(\boldsymbol{\theta})=\left[f_{1} f_{2} f_{3}\right]^{\top}=[R B M]^{\top}$, and the covariance matrix $\mathbf{C}_{\epsilon}(\boldsymbol{\theta})=\operatorname{diag}\left(\sigma_{\epsilon_{R}}^{2} \sigma_{\epsilon_{B}}^{2} \sigma_{\epsilon_{M}}^{2}\right)$, where $\operatorname{diag}(\cdot)$ denotes a diagonal matrix,

$$
\sigma_{\epsilon_{f_{i}}}^{2}=f_{i}^{2} \cdot 10^{\left(-\mathrm{SNR}_{z_{1}} / 10\right)},
$$

where $\mathrm{SNR} z_{1}, \mathrm{SNR} z_{2}$, and $\mathrm{SNR} z_{3}$ represent the $\mathrm{SNRs}$ for the corresponding measurements and are computed as follows:

$$
\mathrm{SNR}_{z_{i}}=10 \log _{10}\left[\frac{f_{i}^{2}}{\sigma_{\epsilon_{f_{i}}}^{2}(\boldsymbol{\theta})}\right]
$$

The Fisher information is a $4 \times 4$ matrix, and $[\mathbf{I}(\theta)]_{11}$ and $[\mathbf{I}(\theta)]_{22}$ are the corresponding elements for $r_{x}$ and $r_{y}$, respectively.

From (A3),

$$
[\mathbf{I}(\theta)]_{11}=R^{-4} \cdot\left[2 r_{x}^{2}+\frac{r_{x}^{2}}{10^{\left(-\mathrm{SNR}_{z_{1}} / 10\right)}}+\frac{r_{y}^{2} \cdot 10^{\left(\mathrm{SNR}_{z_{2}} / 10\right)}}{\left[\arctan 2\left(r_{y}, r_{x}\right)\right]^{2}}+\frac{2 r_{y}^{2}}{\left[\arctan 2\left(r_{y}, r_{x}\right)\right]^{2}}\right]+\frac{10^{\left(\mathrm{SNR}_{z_{3}} / 10\right)}}{r_{x}^{2}}+\frac{2}{r_{x}^{2}},
$$

and

$$
[\mathbf{I}(\theta)]_{22}=R^{-4} \cdot\left[2 r_{y}^{2}+\frac{r_{y}^{2}}{10^{\left(-\mathrm{SNR}_{z_{1}} / 10\right)}}+\frac{r_{x}^{2} \cdot 10^{\left(\mathrm{SNR}_{z_{2}} / 10\right)}}{\left[\arctan \left(\frac{r_{y}}{r_{x}}\right)\right]^{2}}+\frac{2 r_{x}^{2}}{\left[\arctan \left(\frac{r_{y}}{r_{x}}\right)\right]^{2}}\right]+\frac{10^{\left(\mathrm{SNR}_{z_{3}} / 10\right)}}{r_{y}^{2}}+\frac{2}{r_{y}^{2}} .
$$

Similarly, we can compute the remaining elements; subsequently, we obtain CRLB as follows:

$$
\operatorname{Var}\left(\hat{r}_{x}\right) \geq\left[\mathbf{I}^{-1}(\boldsymbol{\theta})\right]_{11}, \quad \operatorname{Var}\left(\hat{r}_{y}\right) \geq\left[\mathbf{I}^{-1}(\boldsymbol{\theta})\right]_{22} .
$$

When using only two measurements of range and bearing, we can obtain the lower bound similarly. For example, $[\mathbf{I}(\theta)]_{11}$ can be obtained by removing the last two terms in (A6). If CRLB for the distance estimation, ie, $\sqrt{r_{x}^{2}+r_{y}^{2}}$ is to be computed, we can use vector parameter CRLB for transformations and is easily derived as follows. ${ }^{27}$ If we define $\boldsymbol{\alpha}=h(\boldsymbol{\theta})=\sqrt{r_{x}^{2}+r_{y}^{2}}$, CRLB is derived as

$$
\operatorname{Var}(\hat{\boldsymbol{\alpha}}) \geq \frac{\partial h(\boldsymbol{\theta})}{\partial \boldsymbol{\theta}} \mathbf{I}^{-1}(\boldsymbol{\theta}) \frac{\partial h(\boldsymbol{\theta})^{\top}}{\partial \boldsymbol{\theta}},
$$

where a Jacobian matrix $\frac{\partial h(\theta)}{\partial \theta}$ is described as

$$
\frac{\partial h(\boldsymbol{\theta})}{\partial \boldsymbol{\theta}}=\left[\begin{array}{llll}
\frac{\partial h(\boldsymbol{\theta})}{\partial \theta_{1}} & \frac{\partial h(\boldsymbol{\theta})}{\partial \theta_{2}} & \ldots & \frac{\partial h(\boldsymbol{\theta})}{\partial \theta_{L}}
\end{array}\right]=\left[\begin{array}{llll}
\frac{r_{x}}{\sqrt{r_{x}^{2}+r_{y}^{2}}} & \frac{r_{y}}{\sqrt{r_{x}^{2}+r_{y}^{2}}} & 0 & 0
\end{array}\right] .
$$

